\title{
A Reproducible MEG/EEG Group Study With the MNE Software: Recommendations, Quality Assessments, and Good Practices
}

\author{
Mainak Jas ${ }^{1}$, Eric Larson ${ }^{2}$, Denis A. Engemann ${ }^{3,4}$, Jaakko Leppäkangas ${ }^{1}$, Samu Taulu ${ }^{2,5}$, \\ Matti Hämäläinen ${ }^{6}$ and Alexandre Gramfort ${ }^{1,3,4 *}$ \\ ${ }^{1}$ Telecom ParisTech, Université Paris-Saclay, Paris, France, ${ }^{2}$ Institute for Learning and Brain Sciences, University of \\ Washington, Seattle, WA, United States, ${ }^{3}$ NeuroSpin, CEA, Université Paris-Saclay, Gif-sur-Yvette, France, ${ }^{4}$ INRIA, Université \\ Paris-Saclay, Saclay, France, ${ }^{5}$ Department of Physics, University of Washington, Seattle, WA, United States, ${ }^{6}$ Athinoula A. \\ Martinos Center for Biomedical Imaging, Massachusetts General Hospital, Harvard Medical School, Charlestown, MA, \\ United States
}

OPEN ACCESS

Edited by:

Pedro Antonio Valdes-Sosa, Clinical Hospital of Chengdu Brain Science Institute, China

Reviewed by:

Stefan Haufe,

Technische Universität Berlin,

Germany

Gareth Barnes,

University College London,

United Kingdom

*Correspondence:

Alexandre Gramfort

alexandre.gramfort@inria.fr

Specialty section:

This article was submitted to

Brain Imaging Methods,

a section of the journal

Frontiers in Neuroscience

Received: 26 December 2017

Accepted: 16 July 2018

Published: 06 August 2018

Citation:

Jas M, Larson E, Engemann DA,

Leppäkangas J, Taulu S,

Hämäläinen M and Gramfort A (2018)

A Reproducible MEG/EEG Group

Study With the MNE Software:

Recommendations, Quality

Assessments, and Good Practices.

Front. Neurosci. 12:530.

doi: 10.3389/fnins.2018.00530
Cognitive neuroscience questions are commonly tested with experiments that involve a cohort of subjects. The cohort can consist of a handful of subjects for small studies to hundreds or thousands of subjects in open datasets. While there exist various online resources to get started with the analysis of magnetoencephalography (MEG) or electroencephalography (EEG) data, such educational materials are usually restricted to the analysis of a single subject. This is in part because data from larger group studies are harder to share, but also analyses of such data often require subject-specific decisions which are hard to document. This work presents the results obtained by the reanalysis of an open dataset from Wakeman and Henson (2015) using the MNE software package. The analysis covers preprocessing steps, quality assurance steps, sensor space analysis of evoked responses, source localization, and statistics in both sensor and source space. Results with possible alternative strategies are presented and discussed at different stages such as the use of high-pass filtering versus baseline correction, tSSS vs. SSS, the use of a minimum norm inverse vs. LCMV beamformer, and the use of univariate or multivariate statistics. This aims to provide a comparative study of different stages of M/EEG analysis pipeline on the same dataset, with open access to all of the scripts necessary to reproduce this analysis.

Keywords: electroencephalography (EEG), magnetoencephalography (MEG), neuroimaging, software, Python, open-source

\section{OVERVIEW}

Magnetoencephalography and electroencephalography (M/EEG) are neuroimaging technologies with a high temporal resolution, which provide non-invasive access to population-level neuronal dynamics on virtually any temporal scale currently considered relevant to cognition. While MEG can recover spatial patterns at a higher signal-to-noise ratio (SNR) and enjoys a more selective cortical resolution than EEG (Baillet, 2017), EEG is more portable and less expensive, and thus supports the study of cognition in a wider range of situations. Processing M/EEG recordings, however, is inherently challenging due to the multi-dimensional nature of the data, the low SNR of brain-related M/EEG signals, and the differences in sensitivity of these measurement techniques. 
This can give rise to complex sequences of data processing steps which demand a high degree of organization from the investigator.

In an effort to address reproducibility issues recently shown to affect neuroimaging studies (Ioannidis, 2005; Carp, 2012a,b; Button et al., 2013), a number of community-led efforts have begun developing data sharing (Poldrack and Gorgolewski, 2017) and data organization (Gorgolewski et al., 2016; Niso et al., 2018) projects. These efforts are necessary first steps, but are not sufficient to solve the problem-they must be complemented by educational tools and guidelines that establish good practices for M/EEG analysis (Gross et al., 2013). However, putting guidelines into practice is not always straightforward, as researchers in the M/EEG community rely on several software packages (Delorme and Makeig, 2004; Dalal et al., 2011; Delorme et al., 2011; Litvak et al., 2011; Oostenveld et al., 2011; Tadel et al., 2011), each of which is different. Even though these packages provide tutorials for single subject data analysis, it is typically left up to the investigator to coordinate and implement multi-subject analyses. Here, we try to address this gap by demonstrating a principled approach to the assembly of group analysis pipelines with publicly available $\operatorname{code}^{1}$ and extensive documentation.

As members and maintainers within the MNE community, we will present analyses that make use of the MNE software suite (Gramfort et al., 2014). Historically, MNE was designed to calculate minimum-norm estimates from M/EEG data, and consisted in a collection of C-routines interfaced through bash shell scripts. Today, the MNE software has been reimplemented in Gramfort et al. (2013a) and transformed into a general purpose toolbox for processing electrophysiology data. Built on top of a rich scientific ecosystem that is open source and free, the MNE software now offers stateof-the-art inverse solvers and tools for preprocessing, timefrequency analysis, machine learning (decoding and encoding), connectivity analysis, statistics, and advanced data visualization. The MNE software, moreover, has become a hub for researchers who use it as a platform to collaboratively develop novel methods or implement and disseminate the latest algorithms from the M/EEG community (Maris and Oostenveld, 2007; Kriegeskorte et al., 2008; Rivet et al., 2009; Gramfort et al., 2010, 2013b; Hauk et al., 2011; Khan and Cohen, 2013; Larson and Lee, 2013; Schurger et al., 2013; Haufe et al., 2014; King and Dehaene, 2014; Engemann and Gramfort, 2015; Smith and Kutas, 2015a,b). With this work, we not only share good practices to facilitate reproducibility, but also present these latest advances in the MNE community which enable automation and quality assessment.

Here, we demonstrate how to use MNE to reanalyze the OpenfMRI dataset ds000117 by Wakeman and Henson (2015). This requires setting the objectives for the data analysis, breaking them down into separate steps and taking a series of decisions on how to handle the data at each of those steps. While there may be several interesting scientific questions that have not yet been addressed on this dataset, here we confine ourselves to the analysis of well-studied time-locked event-related M/EEG

${ }^{1}$ https://github.com/mne-tools/mne-biomag-group-demo components, i.e., event-related fields (ERF) and event-related potentials (ERP). This is motivated by educational purposes to help facilitate comparisons between software packages and address reproducibility concerns. To this end, we will lay out all essential steps from single subject raw M/EEG recordings to group level statistics. Importantly, we will highlight the essential options, motivate our choices and point out important quality control objectives to evaluate the success of the analysis at every step.

We will first analyze the data in sensor space. We will discuss good practices for selecting filter parameters, marking bad data segments, suppressing artifacts, epoching data into time windows of interest, averaging, and doing baseline correction. Next, we turn our attention to source localization: the various steps involved in the process starting from defining a head conductivity model, source space, coregistration of coordinate frames, data whitening, lead field computation, inverse solvers, and transformation of source-space data to a common space. Along the way, we will present various diagnostic visualization techniques that assist quality control at each processing step, such as channel-wise power spectral density (PSD), butterfly plots with spatial colors to facilitate readability, topographic maps, and whitening plots. Finally, we will attempt to distill from our analysis, guiding principles that should facilitate successfully designing other reproducible analyses rather than blindly copying the recipes presented here.

\section{PRELIMINARIES}

In this work, we describe a full pipeline using MNE to analyze the OpenfMRI dataset ds000117 by Wakeman and Henson (2015). The data consist of simultaneous M/EEG recordings from 19 healthy participants performing a visual recognition task. Subjects were presented images of famous, unfamiliar and scrambled faces. The dataset provides a rich context to study different neuroscientific and cognitive questions, such as: Which brain dynamics are characteristic of recognizing familiar as compared to unfamiliar faces? How do commonly studied face-responsive brain regions such as the Superior Temporal Sulcus (STS), the Fusiform Face Area (FFA), and the Occipital Face Area (OFA) interact when processing the familiarity of the face? At the same time, it presents a wellstudied paradigm which can be particularly beneficial for the development of methods related to connectivity and source localization.

\subsection{Data Description}

The subjects participated in 6 runs, each $7.5 \mathrm{~min}$ in duration. In the original study, three subjects were discarded due to excessive artifacts in the data. To produce comparable results, the same subjects are also discarded from the group results in this study. The data were acquired with an Elekta Neuromag Vectorview 306 system consisting of 102 magnetometers and 204 planar gradiometers. In addition, a 70 channel Easycap EEG system was used for recording EEG data simultaneously. 


\subsection{Reading Data}

MNE supports multiple file formats written by M/EEG hardware vendors. Apart from Neuromag FIF files, which are the default storage format, MNE can natively read multiple other formats ranging for MEG data including 4D Neuroimaging BTI, KIT, and CTF, and for EEG data B/EDF, EGI, and EEGLAB set ${ }^{2}$. Despite this heterogeneity of systems, MNE offers a coherent interface to the metadata of the recordings using the so-called measurement info $o^{3}$. Regardless of the input format, all processed files can be saved as FIF files or in the HDF5 format ${ }^{4}$.

MNE can handle multimodal data containing different channel types, the most common being magnetometer, gradiometer, EEG, electrooculogram (EOG), electrocardiogram (ECG), and stimulus trigger channels that encode the stimulation paradigm. MNE also supports electromyogram (EMG), stereotactic EEG (sEEG), and electrocorticography (ECoG), functional near-infrared spectroscopy (fNIRS) or miscellaneous (misc) channel types. Declaring and renaming channel types is a common step in the preparation of M/EEG datasets before analysis. In our case, once the files were read in, some of the channels needed to be renamed and their channel types corrected in the measurement info (see Wakeman and Henson, 2015): the EEG061 and EEG062 electrodes were set as EOG, EEG063 was set as ECG, and EEG064 was set as a miscellaneous channel type as it was a free-floating electrode. If this step is omitted, some preprocessing functions may fall back to potentially less optimal defaults, for example, using the average of the magnetometers instead of the ECG channel when searching for cardiac events.

\section{MEG AND EEG DATA PREPROCESSING}

\subsection{Maxwell Filtering (SSS)}

Neuromag MEG recordings are often preprocessed first using the Signal Space Separation (SSS) method (Taulu, 2006), otherwise known as Maxwell filtering. SSS decomposes the data using multipole moments based on spherical harmonics and removes the component of magnetic field originating from outside the MEG helmet. SSS is therefore useful for removing environmental artifacts, and can also be used to compensate for head movements during the recording. In this study, movement compensation is not strictly necessary as the participants managed to stay predominantly still.

The data provided by OpenfMRI (Poldrack and Gorgolewski, 2017) already contain files processed using the proprietary Elekta software MaxFilter, which is what we use in our analysis for the sake of reproducibility. However, MNE offers an open source reimplementation and extension of SSS as well. Before running SSS, it is crucial that bad channels are marked, as otherwise SSS may spread the artifacts from the bad channels to all other MEG channels in the data. This step is preferably done manually with visual inspection. When using the MNE implementation

\footnotetext{
${ }^{2}$ http://martinos.org/mne/stable/manual/io.html

${ }^{3} \mathrm{http}: / /$ martinos.org/mne/stable/auto_tutorials/plot_info.html

${ }^{4}$ https://support.hdfgroup.org/HDF5/
}

of Maxwell filtering, we reused the list of bad channels available from the Elekta MaxFilter logs in the dataset.

Results comparing raw data, data processed by Elekta MaxFilter, and data processed by the MNE maxwell_filter function are provided in Figure 1. While the unprocessed data do not show a clear evoked response, the Maxwell filtered data do exhibit clear event-related fields with a clear peak around $100 \mathrm{~ms}$ post-stimulus. Note that the results obtained with Elekta implementation and the MNE implementation have minimal differences due to slight differences in computation of component regularization parameters.

\subsubsection{Alternatives}

In principle, SSS can be applied to data acquired with any MEG system providing it has comprehensive sampling (more than about 150 channels). However, so far it has not been tested extensively with other than the 306-channel Neuromag systems. SSS requires relatively high calibration accuracy, and the Neuromag systems are thus carefully calibrated for this purpose. If SSS is not an option, for example due to the lack of fine-calibration information, reasonable noise reduction can be readily obtained from Signal Space Projections (SSP) (Uusitalo and Ilmoniemi, 1997). This intuitively amounts to projecting out spatial patterns of the empty room data covariance matrix using Principal Component Analysis (PCA). In practice, depending on the shielding of the room, the MEG vendor tunes the system so that the raw data file produced contain already some SSP vectors that are meant to be used for removing environmental noise. This number of SSP can vary between installations and go up to a dozen of vectors, especially for magnetometers. Another option in systems containing reference channels (for example, CTF), is to regress out the noise, using so-called "gradient compensation." Indeed, SSS is not so necessary in these systems for suppressing environmental artifacts. However, if the aim is to perform grand averaging, one could still benefit from SSS-based movement compensation to transform the data to a common coordinate space.

\subsubsection{Caveats}

It is important to highlight that after SSS, the magnetometer and gradiometer data are projected from a common lower dimensional SSS coordinate system that typically spans between 64 and 80 dimensions. As a result, both sensor types contain highly similar information, which also modifies the inter-channel correlation structure. This is the reason why MNE will treat them as a single sensor type in many of the analyses that follow.

\subsection{Power Spectral Density (PSD)}

The power spectral density (PSD) estimates for all available data channels provide a convenient way to check for spectral artifacts and, in some cases, bad channels. MNE computes the PSD of raw data using the standard Welch's method (Welch, 1967; Percival and Walden, 1993), whereby the signal for each channel is analyzed over consecutive time segments, with eventually some overlap. Each segment is windowed and then the power of the discrete Fourier transform (DFT) coefficients is computed and averaged over all segments. By making the assumption that each 

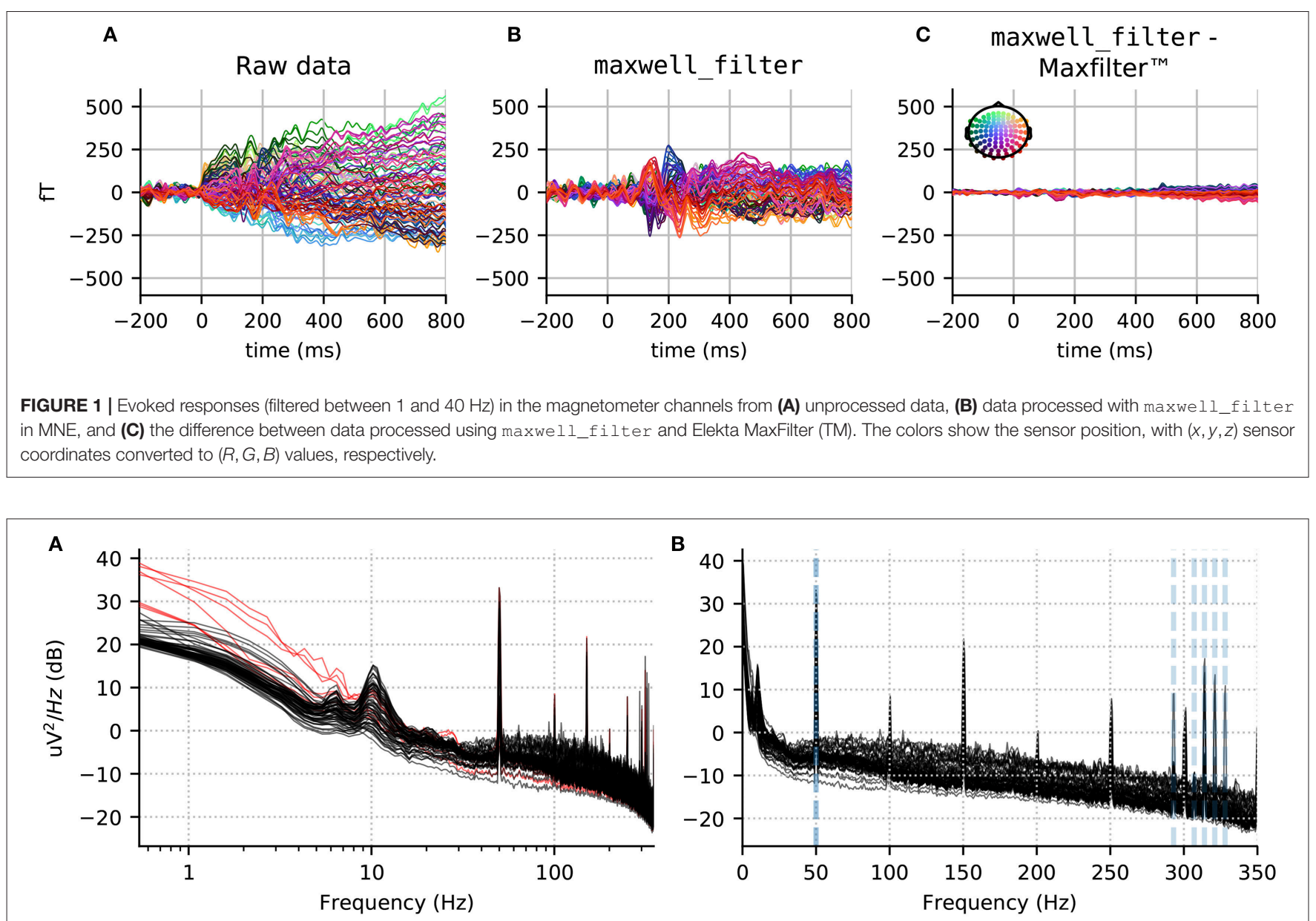

FIGURE 2 | Power spectral density per channel for subject 10, run 02. (A) Log scale for the $x$ axis accentuates low frequency drifts in the data. The red lines show the PSD for the bad channels marked manually and provided to us by Wakeman and Henson (2015). (B) The same data with a linear $\mathrm{X}$-axis scale. Five peaks corresponding to HPI coils around $300 \mathrm{~Hz}$ are visible and marked in gray dotted lines alongside the power line frequency (50 Hz).

of these segments provides a realization of a stationary process, the averaging procedure produces an unbiased estimate of the PSD with reduced noise.

We recommend to visualize channel-wise PSD plots rather than an average across 150 channels, as this facilitates spotting outlier channels. In Figure 2, we show the PSD for the EEG channels in one run for one subject. We use windows of length 8192 samples (about $7.4 \mathrm{~s}$ given the $1.1 \mathrm{kHz}$ sampling rate) with no overlap. Using a power of 2 for the length and no overlap accelerates computations. Using a logarithmic frequency-axis scaling for the PSD enables quality control by facilitating screening for bad channels. In fact, we found that some potentially bad channels (e.g., EEG024 in subject 14 for run 01) were omitted by the authors of Wakeman and Henson (2015), although they are clearly visible in such plots. Concretely we see a few channels with strongly increased low-frequency power below $1 \mathrm{~Hz}$. On the other hand, using a linear frequency-axis scaling, we can convince ourselves easily that the data is unfiltered, as it contains clear peaks from power line at harmonics of $50 \mathrm{~Hz}$, as well as the five Head Position Indicator (HPI) coils used to monitor the head position of the subject, at frequencies of 293 , $307,314,321$, and $328 \mathrm{~Hz}$.

\subsubsection{Alternatives}

The same could have been achieved with the multitaper method (Slepian, 1978; Percival and Walden, 1993), where the data is multiplied element-wise by orthogonal data tapers. However, this method can be an order of magnitude slower than the Welch method for long continuous recordings. The multitaper method is indeed recommended for short data segments. Here we are interested in the PSD for diagnostic purposes on the raw continuous data, and we therefore use the Welch method, a.k.a. averaged periodogram method.

\subsection{Temporal Filtering}

In this study, we focused on event-related brain signals below $40 \mathrm{~Hz}$. We used a zero-phase finite impulse response (FIR) filter using the window design method ("firwin" in $\mathrm{SciPy}^{5}$; Hamming

\footnotetext{
${ }^{5}$ https://docs.scipy.org/doc/
} 
window) to achieve a lowpass with $40 \mathrm{~Hz}$ corner frequency and transition bandwidth of $10 \mathrm{~Hz}$ using a filter length of 363 samples $(0.330 \mathrm{~s})$. Such a filter does not affect ERP signals of interest, attenuates the line frequency of $50 \mathrm{~Hz}$ and all HPI coil frequencies. It also limits the effects of temporal ringing thanks to a wide transition band. Because the low-pass was sufficiently low, we did not employ a notch filter separately. Note that such a choice of filters is not necessarily a good default for all studies of event-related brain responses, as ERFs or ERPs can contain rather high frequencies (see for example Götz et al., 2015).

When filtering, it is important to take into account the frequency response and impulse response of the filter. The default filter used adapts the filter length and transition band size based on the cutoff frequencies, as done in the EEGLAB software (Parks and Burrus, 1987; Ifeachor and Jervis, 2002; Widmann et al., 2015) ${ }^{6}$ Although no default parameters will fit all analysis requirements, MNE chooses parameters that aim to achieve reasonable stop-band attenuation without excessive filter ringing. To illustrate this point, we compare filters across MNE versions using frequency response and impulse response plots in Figure 3. The stop-band attenuation and transition bandwidth in Figures 3A,B are less restricted in the newer versions, which results in less steep attenuation but also less temporal ringing in the impulse response (see Figures 3C,D). It can be seen that the previous default parameters gave rise to stronger filtering artifacts as indicated by higher impulse response amplitudes across the time window.

\subsubsection{Alternatives and Caveats}

If the signal quality is satisfactory, filtering may not be necessary. In the context of this study, we decided to baseline correct our signals rather than high-pass filter them, keeping in mind the ongoing discussion in the community on this topic (Acunzo et al., 2012; Rousselet, 2012; Widmann and Schröger, 2012; Tanner et al., 2015; Maess et al., 2016). Our choice will be motivated in Section 3.7 on baseline correction. Note that the group delay due to filtering would lead to a shift in ERP latency. In the case of linear-phase FIR filters, this can be compensated for. However, in the case of IIR filters with non-linear phase, this is not possible. However, this can be mitigated by using the so-called forwardbackward filtering which involves using the same filter twice: first, with the original signal, and then, with a reversed version of the signal. Another option is to use a peak-preserving filter such as the Savitzky Golay filter. Regardless of the compensation procedure used, it can still affect latencies in time decoding results as discussed in Ramkumar et al. (2013). In this situation, it might be preferable to use filters with shorter impulse response or perform decoding on the unfiltered data.

\subsection{Marking Bad Segments and Channels}

The next step in the pipeline is to remove bad data segments and bad channels. As data have been processed with Maxwell filter, there are no more bad MEG channel at this stage. For the bad EEG channels, we use the ones provided by the original authors.

${ }^{6} \mathrm{https}: / /$ martinos.org/mne/stable/auto_tutorials/plot_artifacts_correction_filtering. html.
To remove bad data segments and bad epochs due to transient artifacts, it is possible in MNE to use the epochs plotter interactively, or to do it via scripting. Either way, the indices of all epochs that are removed from further analysis are logged in the drop log attribute of the epochs objects (see online documentation of the Epochs class ${ }^{7}$ ).

As we are building a reproducible pipeline, here we prefer the scripting route. In MNE, this can be achieved by removing trials whose peak-to-peak amplitude exceeds a certain rejection threshold. Even though this works reasonably well for single subject analysis, it would likely need to be tuned for individual subjects in group studies. Therefore, instead of specifying the thresholds manually, we learn it from the data using the autoreject (global) (Jas et al., 2017) algorithm. Autoreject is an unsupervised algorithm which minimizes the cross-validation error, measured by the Frobenius norm between the average signal of the training set and the median signal of the validation set. Autoreject not only removes trials containing transient jumps in isolated MEG or EEG channels, but also eyeblink artifacts affecting groups of channels in the frontal area. Since we are dealing with visual stimuli, it is preferable to remove the eyeblink trials altogether using the EOG rejection threshold over the stimulus presentation interval rather than suppressing the artifact using a spatial filter such as ICA or SSP. Given the large number of trials at our disposal, we can afford to remove some without affecting the results very much.

For the purpose of group averaging, the bad EEG channels were repaired by spherical spline interpolation (Perrin et al., 1989) so as to have the same set of channels for each subject.

\subsection{Independent Component Analysis (ICA)}

Bad channel or segment removal can correct for spatially and temporally isolated artifacts. However, it does not work well for systematic physiological artifacts that affect multiple sensors. For this purpose, ICA is commonly used (Jung et al., 1998). ICA is a blind source separation technique that maximizes the statistical independence between the components. While PCA only requires orthogonal components, ICA looks for independence for example by looking at higher statistical moments beyond (co)variance. In the context of MEG and EEG analysis, common physiological artifacts have skewed and peaky distributions, hence are easily captured by ICA methods that look for non-Gaussian sources. ICA is therefore popular for removing eye blinks and heart beats, which manifest themselves with prototypical spatial patterns on the sensor array.

In the present study, we use FastICA (Hyvarinen, 1999) to decompose the signal into maximally independent components. We estimate the ICA decomposition on band-pass filtered $(1 \mathrm{~Hz}$ highpass with $1 \mathrm{~Hz}$ transition band, $40 \mathrm{~Hz}$ lowpass with $10 \mathrm{~Hz}$ transition band) data that has been decimated. In practice, to improve the quality of ICA solution, high-pass filtering is often helpful as it can help to minimize violations of the stationarity assumption made by ICA. Likewise, it is recommended to

\footnotetext{
${ }^{7}$ http://martinos.org/mne/stable/auto_tutorials/plot_epoching_and_averaging.
} html 

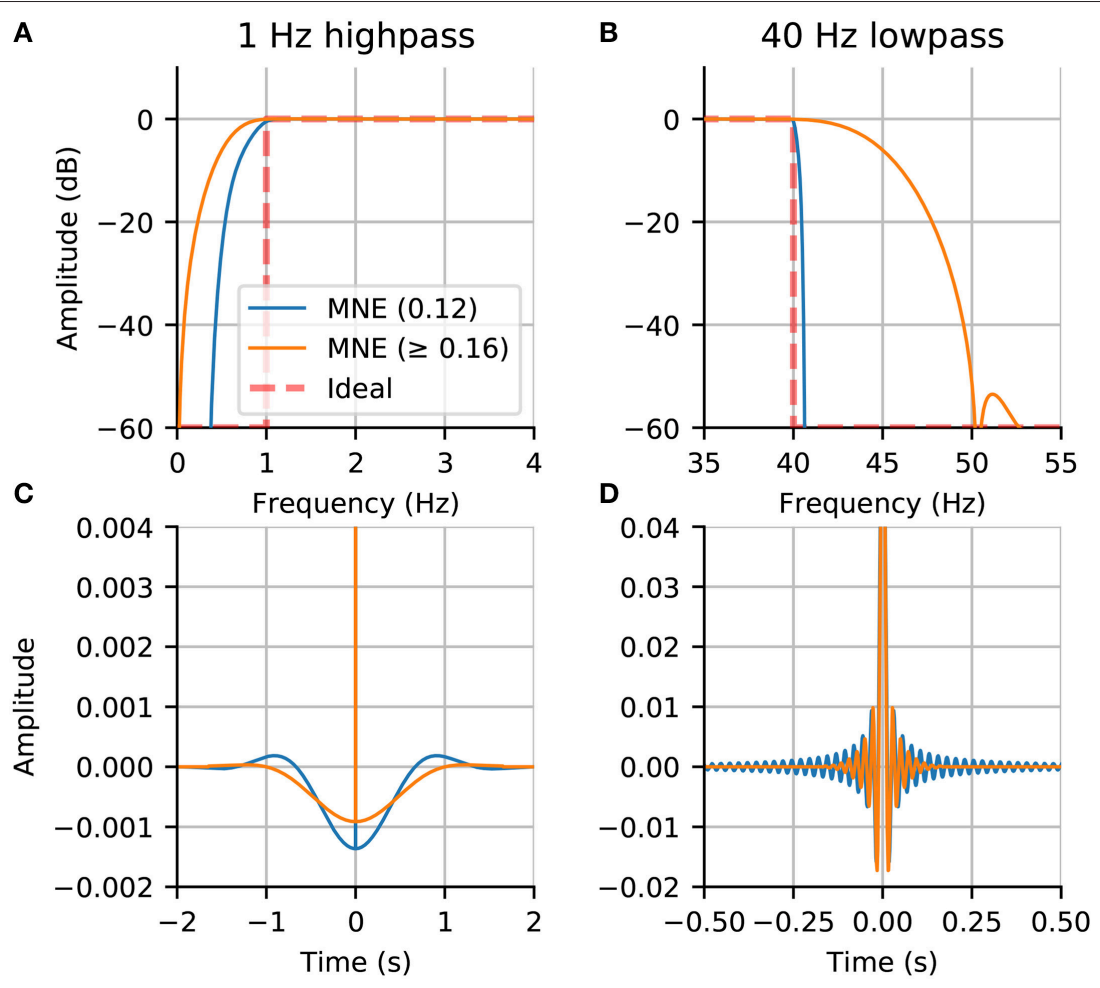

FIGURE 3 | Comparison of filters between new (0.16) and old (0.12) MNE versions: (A) The frequency response of the highpass filter; (B) The frequency response of the lowpass filter; (C) The impulse response of the highpass filter; (D) The impulse response of the lowpass filter. The filters in MNE are now adaptive with trade-offs between frequency attenuation and time domain artifacts that by default adapt based on the chosen low-pass and high-pass frequencies.

exclude data segments containing environmental artifacts with amplitudes higher than the artifacts of interest. Finally, generous decimation can save computation time and memory without affecting the quality of the ICA solution, at least, when it comes to separating physiological artifacts from brain signals. Both measures can be implemented using the reject and decim parameters provided by the ICA fitting routine in MNE. Here we decimated the data by a factor of 11 , and excluded time segments exceeding amplitude ranges of $4000 \times 10^{-13} \mathrm{fT} \mathrm{cm}^{-1}$ and $4 \times 10^{-12} \mathrm{fT}$ on the magnetometers and gradiometers, respectively.

The ICA component corresponding to ECG activity is then identified using cross-trial phase statistics (CTPS) (Dammers et al., 2008) using the default threshold of 0.8 on the Kuiper statistic. Pearson correlations are used to find EOG related components. As ICA is a linear model, the solution can be estimated on continuous raw data and subsequently used to remove the bad components from the epochs or evoked data.

\subsubsection{Alternatives}

MNE also implements CORRMAP (Viola et al., 2009) which is particularly useful when no ECG or EOG channels are available. This approach uses pattern matching of ICA spatial components. Once templates have been manually defined for one subject, similar patterns can be found for the remaining subjects. If ICA is not an option, SSP projections provide a simple and fast alternative. Here, they can be computed from time segments contaminated by the EOG and ECG artifacts and commonly the first 1 to 2 components are projected out. In our experience, SSP is less precise in separating artifacts from brain components than ICA for the reasons mentioned above, yet, often good enough for a wide class of data analysis scenarios. For analysis of single EEG sensors, multivariate methods cannot be applied. Computing the residuals of a linear regression from the ECG sensor on the EEG is an option in this case.

\subsubsection{Caveats}

Before blindly applying ICA, it is recommended to estimate the amount of contamination of the MEG and EEG signals. This can be easily achieved by detecting artifact events and epoching and averaging the data accordingly. If, for example, the amplitude range of the average ECG artifact is close to the amplitude range of the brain signals and only few events occur, chances are low to estimate clear cut ECG components using ICA. However, in this case the contamination by ECG is low and therefore no advanced artifact suppression is needed. Second, there is a tradeoff between processing time and accuracy. For many analyses, mitigating the artifact contamination by a significant proportion is sufficient and methods like SSP are a reasonable choice. In certain decoding analyses, such preprocessing considerations may have little relevance if any for the final classification results. Indeed, the combination of supervised and multivariate decoding 
algorithms allows to extract the signals of interest directly in one step.

\subsection{Epoching}

In event-related $\mathrm{M} / \mathrm{EEG}$ studies, a trigger channel (in this data STI101) contains binary-coded trigger pulses to mark the onset/offset of events. These pulses can be automatically extracted from the data during analysis and the values on the trigger channel are mapped to the event IDs. MNE offers the possibility to extract events when the signal in the trigger channel increases, decreases, or both. It also allows the construction of binary masks to facilitate selecting only the desired events. We masked out the higher order bits in the trigger channel when extracting the events as these corresponded to key presses. After extraction, events can be freely manipulated or created as necessary by the user, as they only require (i) the sample number, and (ii) some integer code relevant for the experiment or analysis.

As a next step, we extracted segments of data from the continuous recording around these events and stored them as single trials, which are also called epochs, in MNE. The Epochs object can store data for multiple events and the user can select a subset of these as epochs [event_id] ${ }^{8}$. Moreover, MNE offers the possibility for the user to define a hierarchy of events by using tags (similar in flavor to hierarchical event descriptors by Bigdely-Shamlo et al., 2013). This is done using event_id which is a dictionary of key-value pairs with keys being the tags separated by a forward slash (/) and values being the trigger $\operatorname{codes}^{9}$. For the paradigm used in this study we used:

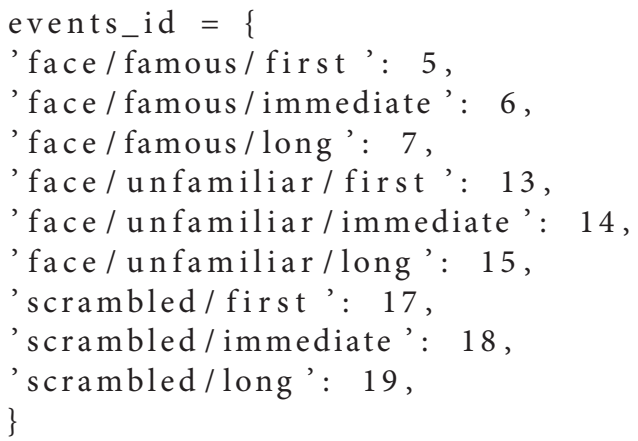

At the highest level of hierarchy are "face" and "scrambled." A "face" can be "famous" or "unfamiliar." And a famous face can be "first," "immediate," or "long" (This distinction between the three categories of famous faces was not used in our analysis). Later on, accessing all the epochs related to the "face" condition is straightforward, as one only needs to use epochs ['face' ] and MNE internally pools all the sub-conditions together. Finally, the epochs were constructed starting $200 \mathrm{~ms}$ before stimulus onset and ending $2900 \mathrm{~ms}$ after (the earliest possible time of the next stimulus onset).

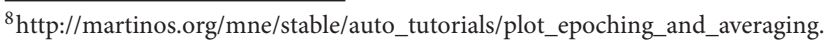
html

${ }^{9} \mathrm{http} / / /$ martinos.org/mne/stable/auto_tutorials/plot_object_epochs.html
}

\subsection{Baseline Correction}

It is common practice to use baseline correction so that any constant offsets in the baseline are removed. Highpass filtering achieves similar results by eliminating the lowfrequency components in the data. However, when using baseline correction, the low frequency drifts present in the data are not attenuated. Thus it is useful to examine long time-courses of the data, if possible, to determine if low-frequency drifts are present. The difference between the two approaches can be seen in Figure 4. The evoked responses in the figure are acrosstrial averages for the famous face condition. If a maximum time of approximately one second were used, a simple baseline correction would appear to produce an undesired "fanning" in the later responses. Indeed one can observe in Figure 4A that at one second post-stimulus, the channels still significantly deviate from zero. However, by extending the time window much longer (here to $2.9 \mathrm{~s}$ ) we can see that the signals do mostly return to the baseline level.

\subsubsection{Caveats and Alternatives}

With highpass filter at $1 \mathrm{~Hz}$ (and $1 \mathrm{~Hz}$ transition band), the signal returns to the baseline level much sooner. Note also the similarities between Figures 4B,C, illustrating how using temporal version of the SSS algorithm (tSSS) acts implicitly as a high-pass filter. For tSSS, we use a buffer size of length $1 \mathrm{~s}$ and a correlation limit of 0.95 to reject overlapping inner/outer signals. However, these high-passing effects come at the expense of distorting the sustained responses. We will thus focus on analyses that utilize the baseline-corrected data here.

\section{SENSOR SPACE ANALYSIS}

An important step in analyzing data at single-subject and group levels is sensor-space analysis. Here we show how several different techniques can be employed to understand the data.

\subsection{Group Average}

A classical step in group studies is known as "grand averaging" (Delorme et al., 2015). It is particularly common for EEG studies and it consists in averaging ERPs across all subjects in the study. As not all subjects have generally the same good channels, this step is commonly preceded by an interpolation step to make sure data are available for all channels and for all subjects. Note that grand averaging is more common for EEG than for MEG, as MEG tends to produce more spatially resolved topographies that may not survive averaging due to signal cancelations.

The grand average of the 16 subjects for one EEG sensor (EEG065) is presented in Figure 5. We selected this channel to compare with the figure proposed by Wakeman and Henson (2015). We present the grand average for the "scrambled," "famous," and "unfamiliar" conditions using a high-pass filter (cf. Section 3.7), and baseline corrected using prestimulus data. This figure replicates the results in (Wakeman and Henson, 2015). We can see the early difference between faces, familiar or unfamiliar, and scrambled faces around $170 \mathrm{~ms}$. We can also notice a difference in the late responses between the two 

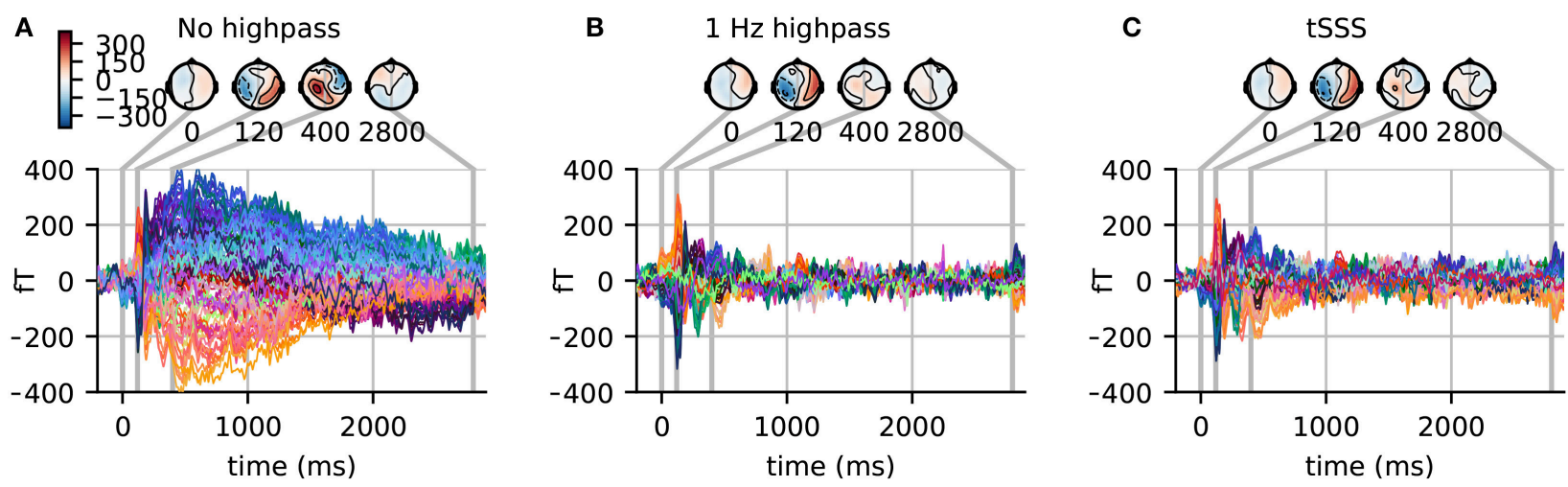

FIGURE 4 | (A) Evoked response in magnetometers for subject 3 with baseline correction. Note how signals tend toward the baseline late in the epochs (where the rightmost time point, $2.9 \mathrm{~s}$, is the earliest possible start time for the next stimulus). (B) The highpass filtered version of the signal and (C) the signal processed with temporal SSS (tSSS). Both reduce the magnitude of the slow and late sustained responses shown in (A).
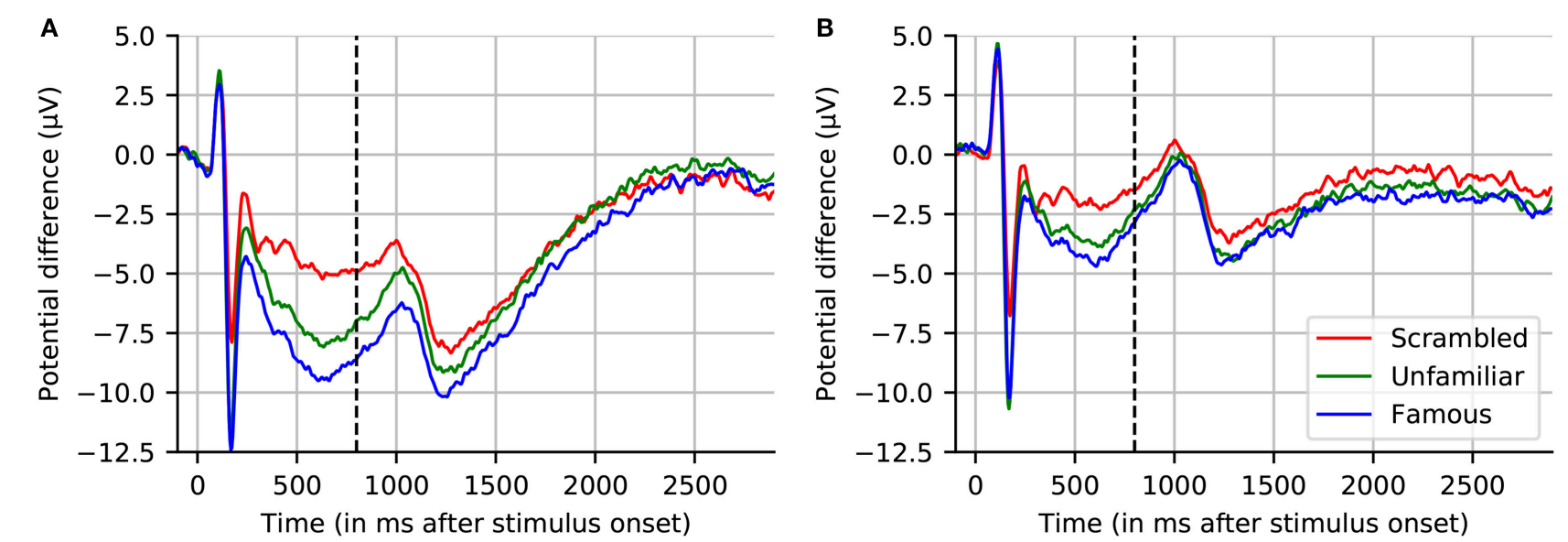

FIGURE 5 | Grand averaged evoked response across 16 subjects for channel EEG065. (A) No highpass filter. (B) Highpass filtered at 1.0 Hz. Note that, similar to (A), the results reported by Wakeman and Henson (2015) (dashed line at 800 ms indicates where their plot stopped) show large drifts, but these return to near-baseline levels toward the end of a sufficiently long interval (here, $2.9 \mathrm{~s}$ ) even without applying a highpass filter.

conditions "unfamiliar" and "famous." However, the effect is smaller when using high-pass filtering, as it corrects for the slow drifts.

\subsubsection{Caveats}

For MEG, the grand average may wash out effects or induce spurious patterns due to misalignment between head positions. SSS can be used to align subjects in one common coordinate systems.

\subsection{Contrasting Conditions}

In this study, we consider pairs of conditions. At the group level, the statistical test will consider the subjects as observations. For each subject, before the group level test, we need to compute the contrast between conditions. In the sensor space analyses, we obtain these contrasts by subtracting the evoked responses of each condition, e.g., subtracting the average of the faces epochs to the average of the scrambled face epochs. In source space (see
Section 5 if unfamiliar with source space analysis), we compute the difference between the source estimates (which take the norm on a vertex-by-vertex basis). Such differences do not take into account the number of trials used to compute the evoked responses-in other words, each condition is weighted equally. In the following we compare not only "faces" against "scrambled faces," but also "famous faces" against "unfamiliar faces."

\subsubsection{Caveats}

Although it is quite standard in EEG pipelines to subtract conditions without taking into account the number of trials, this needs to be carefully thought through. When subtracting two evoked responses with substantially different number of trials, one evoked response will be more noisy, and therefore the subtraction will have a noise level that should be properly estimated. This computation of adjusted SNR and noise variance is done by the mne.combine_evoked function, which was used in this study with the option weights='equal'. By 
using this option, if one considers a dataset with 100 trials in condition $\mathrm{A}$ and 10 trials in condition $\mathrm{B}$, by weighting the averages across each condition equally, a single trial from condition A is effectively weighted 10 times less than a single trial from condition $\mathrm{B}$. This has the effect of amplifying noise from condition $\mathrm{B}$ but maintaining the amplitude of the underlying signal. However, this function can also be used with option weight $s=$ 'nave'. In that case, the number of trials averaged together will be taken into account in the contrast so that each trial, in either condition, will have the same weight. In this case, with the above example, the individual trials from the first condition will effectively be weighted the same way as trials from the second condition, which means that any underlying responses in condition A will be 10 times larger than those in condition B. This has been historically recommended by the MNE software for source estimation, but can be problematic when working with certain experimental protocols (for example, oddball tasks) which, by design, produce many more trials for one condition than for the other.

\subsection{Cluster Statistics}

To compare our conditions of interest, here we use a nonparametric clustering statistical procedure as described by Maris and Oostenveld (2007). This method works by combining neighboring values that are likely to be correlated (e.g., neighboring time instants or spatial locations) to reduce the problem of multiple comparisons. For each feature, a t-statistic is first computed, these are thresholded, and combined based on sufficient proximity to form clusters. The t-values for each cluster are then summed (or number of spatio-temporal points counted) to compute the mass (or size) of each cluster, which serves as the cluster-level statistic. Next, we need to know if the distribution of data in our two conditions-here measured using cluster masses-differ from one another significantly more than what would be obtained by chance. For this purpose, we generate a null distribution from the data by randomly swapping our conditions for each subject according to exchangeability under our null hypothesis. In our paired contrast, this exchangeability is equivalent to changing the sign of the subtracted evoked data. For each permutation, clusters are formed and only the maximal cluster mass is retained. The distribution of these maximal cluster masses gives us our null data distribution, from which we can compute the probability of observing each cluster under the null hypothesis. This gives us a control of the family-wise error rate (FWER), a.k.a. type 1 error, when reporting a significant cluster in our comparison of two conditions.

Running this nonparametric permutation test on the single sensor EEG065 (also used by Wakeman and Henson, 2015) allowed us to reject our null hypothesis $(p<0.01)$ due to two temporal clusters (cf. Figure 6). To threshold the original t-values and estimate the candidate clusters, we used an initial threshold corresponding to a probability of 0.001 with a twosided paired $t$-test. A first cluster appears around the same time as the evoked response, and the other captures the late effects. Running another statistical test, this time incorporating the spatial distribution of the sensors into the clustering procedure, yields one spatiotemporal cluster $(p<0.05)$ as shown in Figure 7.

\subsubsection{Alternatives and Caveats}

It is important to note that this clustering permutation test does not provide feature-wise (vertex, sensor, time point, etc.) but cluster-level inference. This is because the test statistic is the cluster mass. When inspecting a significant cluster, no conclusion can be drawn on which time point or location was more important. A computationally more expensive alternative is the so-called threshold-free cluster enhancement (TFCE) method
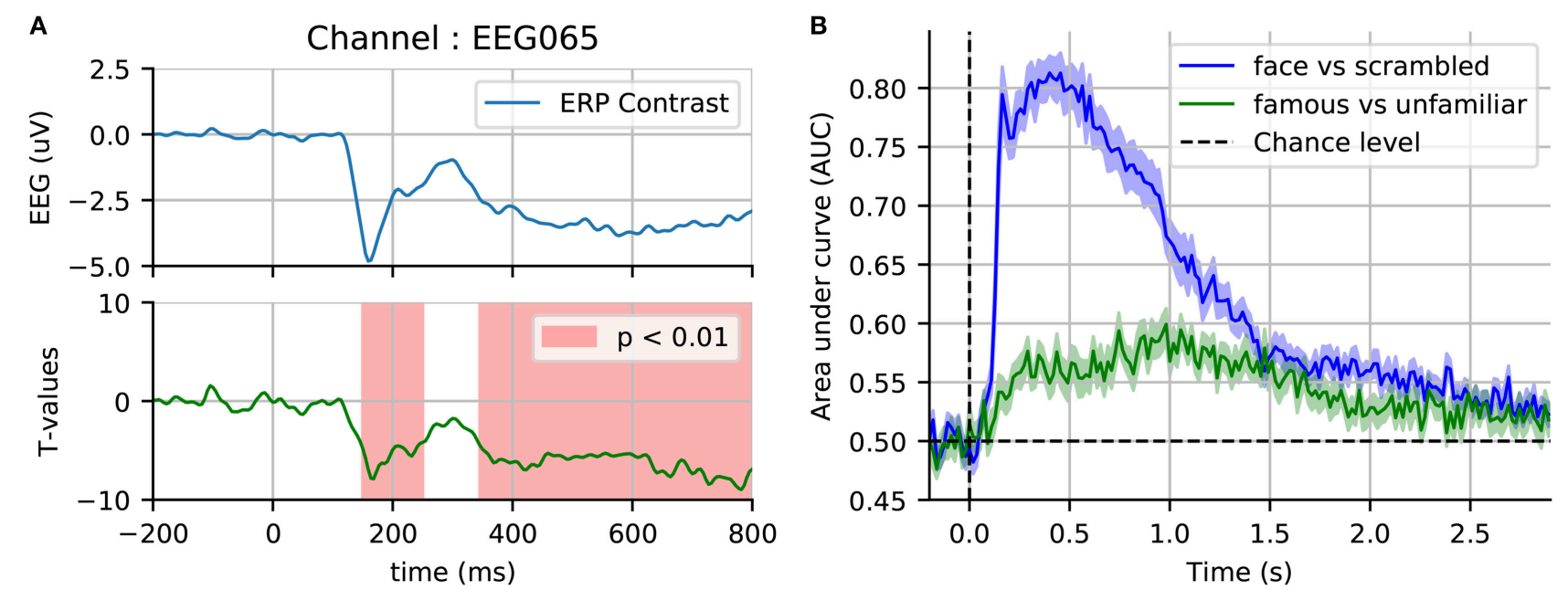

FIGURE 6 | Sensor space statistics. (A) A single sensor (EEG065) with temporal clustering statistics. The clustering is based on selecting consecutive time samples that have exceeded the initial paired $t$-test threshold (0.001), and finding clusters that exceed the mass expected by chance according to exchangability under the null hypothesis ( $p<0.01$, shaded areas). (B) Cross-validation score of time-by-time decoding. As opposed to a cluster statistic, time decoding is a multivariate method which pools together the signal from different sensors to find discriminative time points between two conditions. 

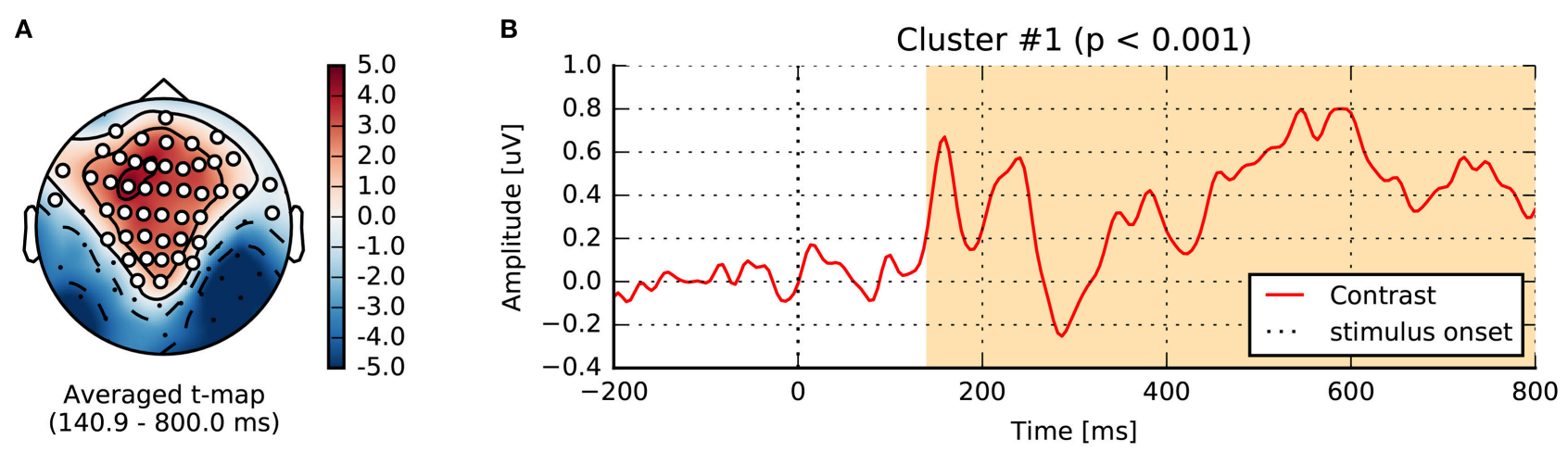

FIGURE 7 | Spatiotemporal cluster statistics on the EEG sensors. (A) Topographic map of the t-statistic. (B) Average over the sensors that were part of the significant cluster.

which provides feature-level inference and, moreover, mitigates the problem of having to set the initial threshold on the t-values to define clusters (Smith and Nichols, 2009). When strong a priori hypotheses exist considering few regions of interest in either time, frequency or space can be a viable alternative. In that case, the multiple comparisons problem may be readily alleviated by more conventional measures, such as false discovery rates (FDR) (Genovese et al., 2002).

\subsection{Time Decoding-Multivariate Analysis}

As an alternative to mass-univariate analysis, a event-related brain dynamics can studied using a multivariate decoding approach (Ramkumar et al., 2013; King and Dehaene, 2014). Here, a pattern classifier, often a linear model (e.g., logistic regression) is trained to discriminate between two conditions: "face" vs. "scrambled," and also "famous faces" vs. "unfamiliar faces." The classifier is trained on single trials, time-point by time-point on MEG data for all the available 306 sensors except those marked as bad. The prediction success can then be assessed with cross-validation at every instant, yielding an intuitive display of the temporal evolution of discrimination success. In Figure 6B, we display such cross-validation time-series averaged across the 16 subjects. As anticipated, discriminating between faces and scrambled faces is much easier than discriminating between "famous" and "unfamiliar" faces, based on information in early components in the first second after stimulus-onset.

For performance evaluation, we use is area under the receiver operating characteristic curve (ROC-AUC), as it is a metric that is insensitive to class imbalance (i.e., differing numbers of trials) therefore allowing us to average across subjects, and also to compare the two classification problems (faces vs. scrambled and familiar vs. unfamiliar). Results on the faces vs. scrambled conditions show that time-resolved decoding reveals decoding accuracy greater than chance around the same time intervals as the non-parametric cluster statistic. The effect although appears here quite sustained over time. Results on familiar $v s$. unfamiliar conditions are also above chance from 200 to $300 \mathrm{~ms}$, however the best decoding performance emerges later for this contrast. This more subtle effect even peaks after $800 \mathrm{~ms}$, which exceeds the time window investigated in the original study.

\subsubsection{Caveats}

Often, it is the case that classification performance is not affected by the presence of artifacts. However, in the case of systematic biases in the artifacts, it is worth noting that a good classification performance can result from two different reasons. Ideally of course, the classifier is able to project away the artifacts and find discriminating directions orthogonal to the artifact space. However, sometimes the artifacts may be predictive themselves (e.g., eye blinks in a visual detection paradigm) and thus be correlated with the discriminative brain signals. In this case, while there is not much that can be done to prevent artifacts from contributing to the classification, it is instructive to check the information maps to understand what features drove the classification (Haufe et al., 2014). This way, one can rule out classification performance being a result of artifacts such as eye-blinks rather than neural activity.

\section{SOURCE RECONSTRUCTION}

The MNE software relies on the FreeSurfer package (Dale et al., 1999; Fischl et al., 1999) for the processing of anatomical MRI images. This automatic procedure is run using the command recon-all on the T1 MRI of each subject. This provides many useful pieces of information, but the most critical here are the cortical reconstruction (a high resolution triangulation of the interface between the white and the gray matter) and the inner skull surface.

For inverse source reconstruction and beamforming, we must first compute the forward solution, often called a gain or lead field matrix (Mosher et al., 1999). Computing the gain matrix, which is a linear operator, requires having a so-called source space of dipole locations, a conductor model for the head, and the sensor locations relative to those dipoles. This latter requirement in practice means putting in the same coordinate system the MRI (where the source space and conductor model are defined), the head (where the EEG electrodes are digitized), and the MEG device (where the MEG sensors are defined). This step is commonly referred to as coregistration. We will cover each of these steps below. 


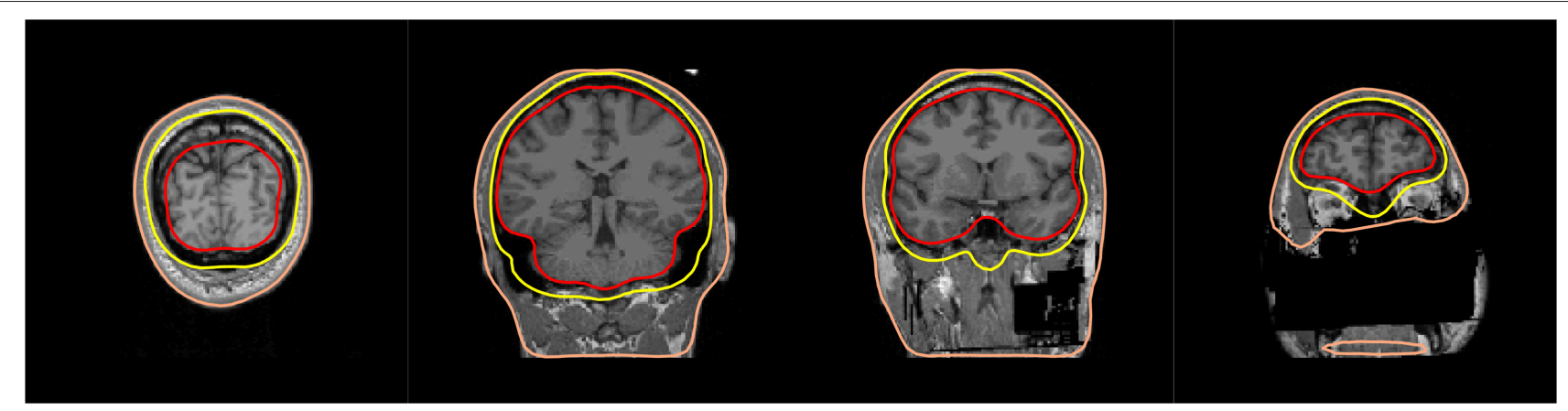

FIGURE 8 | BEM surfaces on flash MRI images. The inner skull, outer skull, and outer skin are outlined in color.

\subsection{Source Space}

As we expect most of our activations of interest to be due to cortical currents (Dale et al., 2000), we position the candidate dipoles on the cortical mantel. It is worth noting that for certain datasets, this assumption may not necessarily be true. For instance, in the case of brainstem or cerebellum activations one could add these structures to the model as proposed by the MNE software. We chose a cortical source space obtained by recursively subdividing the faces of an octahedron six times (oct6) for both the left and right hemispheres. This leads, for each subject, to a total of 8196 dipoles evenly spaced on the cortical surface (See Figure 6 in Gramfort et al., 2014).

\subsection{Head Conductivity Model}

MNE can use simple spherical conductor models but when the MRI of subjects are available, the recommended approach is to use a piecewise-constant conductivity model of the head. Tissue conductivities are defined for each region inside and between the segmented interfaces forming the inner skull, outer skull and the outer skin. It corresponds to a so-called three layer model, however a single layer is possible when using only MEG data. The default electrical conductivities used by MNE are $0.3 \mathrm{~S} / \mathrm{m}$ for the brain and the scalp, and $0.006 \mathrm{~S} / \mathrm{m}$ for the skull, i.e., the conductivity of the skull is assumed to be $1 / 50$ of that of the brain and the scalp. With such a head model, Maxwell equations are solved with a boundary element model (BEM).

In addition to the T1 MRI image, fast low-angle shot (FLASH) images are provided in the present dataset. Such MRI images allow to automatically extract precise surfaces for the inner skull and outer skull. Note that in the absence of FLASH images, MNE offers a somewhat less accurate solution based on the watershed algorithm. One output of the MNE automatic BEM surface extraction is presented in Figure 8. It contains the three surfaces needed for the computation of the EEG gain matrix. In our results shown here, we used only the MEG data for source reconstruction, and consequently only made use of the inner skull surface in a one-layer model. As MRIs shared here are defaced, outer skull and scalp surfaces are anyway quite wrong, so we considered it satisfactory to only use the inner skull surface.

Quality insurance at this stage consists in checking that the three surfaces do not intersect with each other and that they follow the interfaces between the brain, the skull and the skin. A slice-by-slice visual inspection of approximate alignment is best and is conveniently proposed by MNE BEM plotting function that outputs a figure as presented in Figure 8.

Here, as the MRIs shared in this dataset were anonymized, the outer skin surface obtained automatically using Freesurfer intersected with the outer skull surface for most subjects. However, this is rarely observed with non defaced T1 MRI images.

\subsection{Coregistration}

In order to compute the gain matrix, the sensor locations (and normals), head model, and source space must be defined in the same coordinate system. In practice, this means that the BEM surfaces and source space (which are defined in MRI coordinates) must be coregistered with the EEG sensors, which are digitized in the Neuromag head coordinate frame (defined by the digitized nasion, LPA, and RPA). The MEG sensor locations and normals are defined in the MEG device coordinate frame. Typically, the MEG-to-head transformation is determined during acquisition using head position indicator (HPI) coils (or redefined using head position transformation using Maxwell filtering), so MEG sensors can be easily transformed to head coordinates. The transformation between the MRI and head coordinate frames is typically estimated by identifying corresponding points in the head and MRI coordinate systems, and then aligning them.

The most common points used to provide an initial alignment are the fiducial landmarks that define the Neuromag head coordinate frame. They consist of the nasion and two preauricular points which are digitized during acquisition, and are then also identified by offline visual inspection on the MRI images. Additional digitization points on the head surface can also be used to better adjust the coregistration. In this study, on average, 135 digitization points were available per subject. The transformation, which consists of a rotation matrix and a translation vector, is then typically saved to a small file, also called trans file, and later used to compute the forward solution.

For quality insurance, MNE offers a simple function to visualize the result of the coregistration. Figure 9 shows one example obtained with this function with the defaced, lowresolution MRI head surface. As here the MRI were defaced, 


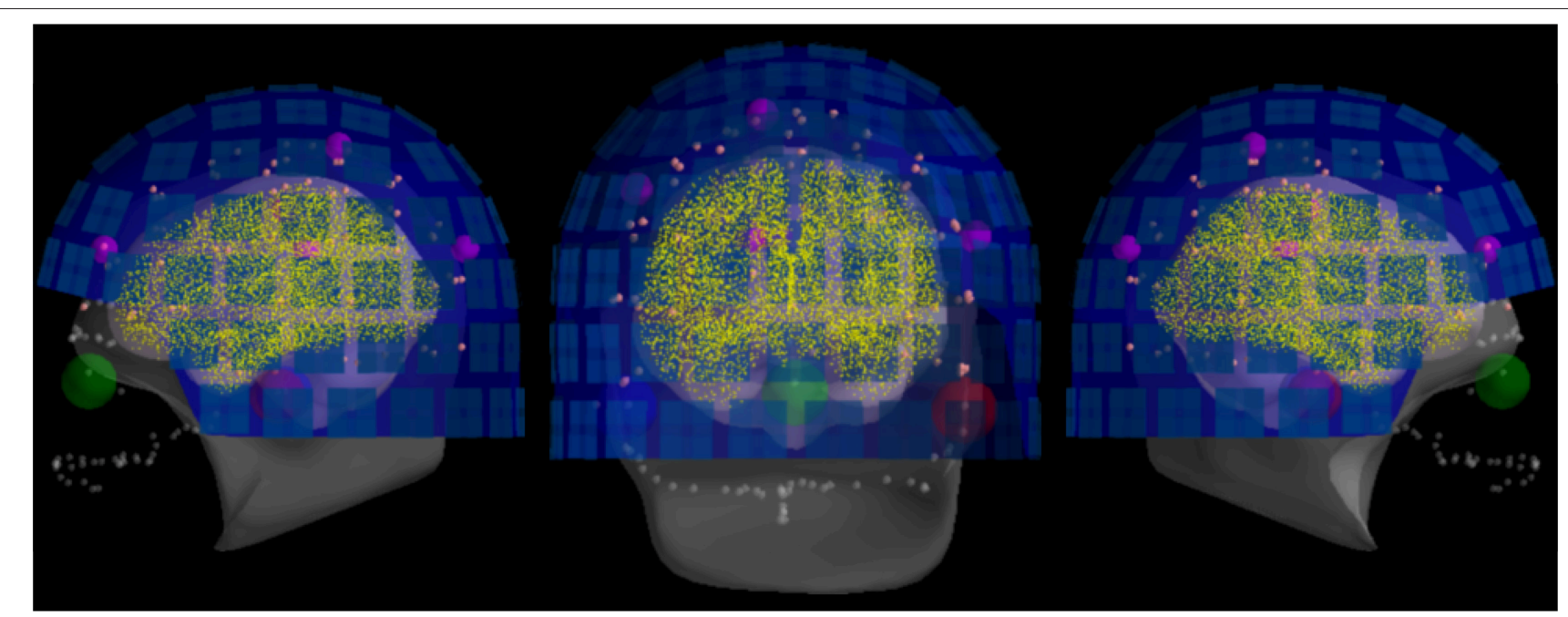

FIGURE 9 | The result of head-to-MRI (and MEG-to-head) transformations with inner skull and outer skin surfaces for one subject. Note that the MEG helmet is well-aligned with the digitization points. The digitized fiducial points are shown with large dots, EEG electrodes with small pink dots, and extra head digitization points with small gray dots. Note that the anonymization of the MRI produces a mismatch between digitized points and outer skin surface at the front of the head.

many important digitization points close to the nose where useless. To reduce the risk of bad coregistration due to defaced MRI images, we used the trans files kindly provided by the original authors.

\subsection{Covariance Estimation and Whitening}

As inverse solvers typically assume Gaussian noise distribution on the sensors with an identity covariance matrix, a whitening step is first necessary (Engemann and Gramfort, 2015). M/EEG signals are indeed highly spatially correlated. Whitening also allows integration of data from different channel types that can have different units and signal amplitudes which differ by orders of magnitudes (cf. planar gradiometers, axial magnetometers, and EEG electrodes). To whiten the data, one must provide an estimate of the spatial noise covariance matrix. This can be computed from empty-room recordings for MEG or prestimulus periods (Gramfort et al., 2014). Here, we followed the approach proposed by Engemann and Gramfort (2015), which consists in picking the best model and estimating the best regularization parameters by computing the Gaussian loglikelihood of left-out data (i.e., a cross-validation procedure). Such an approach has been shown to be particularly robust for scenarios where a limited number of samples is available for covariance estimation.

In this analysis, the noise covariance is estimated from the $200 \mathrm{~ms}$ of data before stimulus presentation. During this period, only a fixation color is visible at the center of the screen. Given this covariance matrix and the gain matrix, one can assemble the inverse operator to compute the MNE or dSPM solutions (Dale et al., 2000).

The quality of the covariance estimation and whitening can have a significant impact on the source localization results. The rank-adjusted global field power (GFP) has been proposed by Engemann and Gramfort (2015) as a measure that can be used to check the quality of the whitening. It is defined as GFP= $\sum_{i} x_{i}^{2} / P$ where $P$ is the rank of the data and $x_{i}$ is the signal in the $i$ th sensor at a time instant. The GFP being a normalized sum of Gaussian random variables with an identity covariance matrix, it follows a $\chi^{2}$ distribution with an expected value of 1 . What is not captured by our noise model, e.g., actual brain signals, thereof will pop out in the whitened domain. To understand this better, we show some whitened data and the GFP in Figure 10. If the Gaussian assumption has not been violated, we expect the whitened data to contain $95 \%$ of the signal within the range of -1.96 and 1.96 , which we mark in dotted red lines. The baseline period, where we estimated our noise covariance from, appears to satisfy this assumption. Consequently, the GFP is also 1 during this period. One can observe a strong increase in the GFP just after the stimulus onset, and that it returns slowly to 1 at the end of the time interval. Such a diagnostic plot can in fact be considered essential for quality assurance before computing source estimates. This has as consequence that what appears in the source estimates depends on our noise model. For instance, using a noise covariance obtained from empty room recordings would suggest the presence of "interesting" signals, simply because it contains brain signals that are fundamentally different from the empty room noise.

For the LCMV beamformer, we also need to estimate a signal covariance. For this we use the $30 \mathrm{~ms}$ to $300 \mathrm{~ms}$ window after the stimulus onset. The data covariance is again regularized automatically following (Engemann and Gramfort, 2015) and is motivated by the results from Woolrich et al. (2011) and Engemann et al. (2015).

\subsubsection{Caveats}

If empty-room data are used to whiten processed signals, one must make sure that the obtained noise covariance matrix 

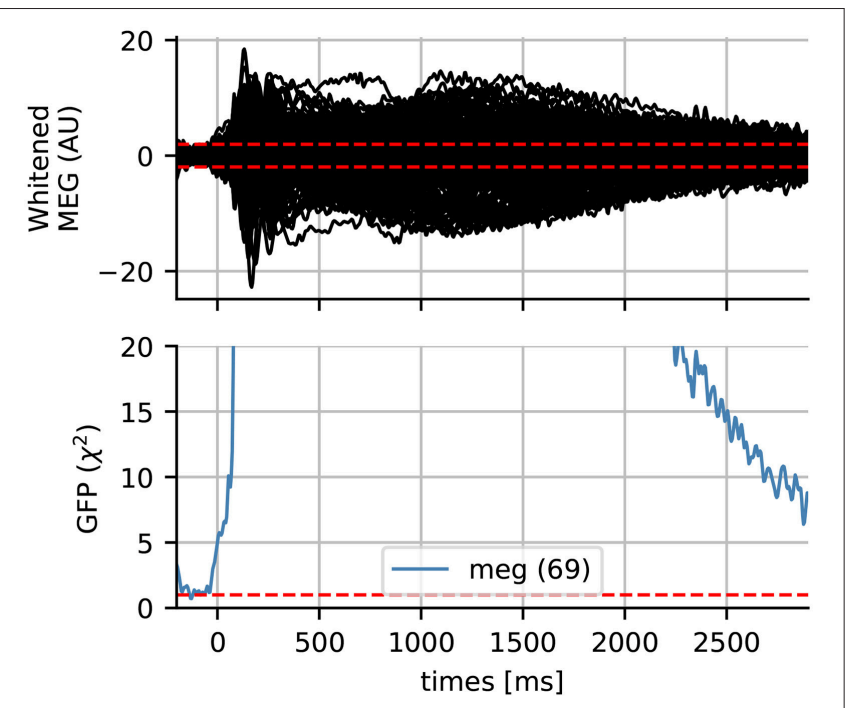

FIGURE 10 | Whitened MEG data for subject 4 and the global field power (GFP) which follows a $\chi^{2}$ distribution if the data is assumed Gaussian. The dotted horizontal red lines represent the expected GFP during the baseline for Gaussian data. Here the data slowly return to baseline at the end of the epoch.

corresponds to the processed data rather than to the original empty-room data. This is done by processing the empty-room data with exactly the same algorithm and the same parameters as the actual data to be analyzed. For example if SSS, SSP, or ICA are applied on processed data, it should be applied to empty room data before estimating the noise covariance. Concretely, SSP vectors and ICA components projected out from the data of interest should also be projected out from the empty room data. SSS should be performed with identical parameters. Also note that magnetometers and gradiometers are whitened jointly. Moreover, if SSS was applied, the display of whitening treats magnetometers and gradiometers as one channel-type. For proper assessment of whitening, a correct assessment of the spatial degrees of freedom is necessary. The number of SSS dimensions is commonly a good estimate for the degrees of freedom. When movement compensation was applied, the estimated data rank maybe unreliable and suggest too many independent dimensions in the data. Even the actual number of SSS components can be misleading in such circumstances. It is then advisable to inspect the eigenvalue spectrum of the covariance matrix manually and specify the degrees of freedom manually using the rank parameter.

\subsection{Inverse Solvers and Beamforming}

The goal of an inverse solver is to estimate the locations and the time courses of the sources that have produced the data. While the data $\mathbf{M}$ can be expressed linearly from the sources $\mathbf{X}$ given the gain matrix $\mathbf{G}, \mathbf{M} \approx \mathbf{G X}$, the problem is ill-posed. Indeed $\mathbf{G}$ has many more columns than rows. This means that there are more unknown variables (brain sources) than the number of measured values (M/EEG sensors) at each time point. This also implies that the solution of the inverse problem is not unique.

For this reason, many inverse solvers have been proposed in the past ranging from dipole fits (Scherg and Von Cramon, 1985; Mosher et al., 1992), minimum norm estimates (MNE) (Hämäläinen and Ilmoniemi, 1984), and scanning methods such as RAP-MUSIC or beamformers such as LCMV and DICS (Van Veen et al., 1997; Gross et al., 2001; Sekihara et al., 2005). There is therefore no absolute perfect inverse solver, although some are more adapted than others depending on the data. Certain solvers are adapted to evoked data for which one can assume a few set of focal sources. Certain methods also provide source amplitudes in a proper unit, which is nAm for electrical current dipoles, such as MNE, MxNE Gramfort et al. (2013b) or dipole fits. Other methods yield spatially normalized statistical maps such as dSPM (Dale et al., 2000) or LCMV combined with neural activation index (NAI) filter normalization (Van Veen et al., 1997).

Given the important usage of dSPM and the LCMV beamformer in the cognitive neuroscience literature, we wanted to investigate how much using one of these two most commonly used methods was affecting the source localization results. The dSPM solution was computed with MNE default values: loose orientation of 0.2 , depth weighting (Lin et al., 2006) of 0.8 , and SNR value of 3. The LCMV used was a vector beamformer with unit-noise-gain normalization (Sekihara et al., 2005) as implemented in MNE 0.15. No specific regularization was used in the beamformer filter estimation.

\subsection{Group Source Reconstruction}

To analyze data at the group level, some form of data normalization is necessary, whereby data from all subjects is transformed to a common space in a manner that helps compensate for inter-subject differences. This procedure, called morphing by the MNE software, exploits the FreeSurfer spherical coordinate system defined for each hemisphere (Dale et al., 1999; Fischl et al., 1999). In our analysis, the data are morphed to the standard FreeSurfer average subject named fsaverage. The morphing procedure is performed in three steps. First, the subsampled data defined on the high resolution surface are spread to neighboring vertices using an isotropic diffusion process. Next, registration is used to interpolate the data on the average surface. And finally, the data defined on the average surface is subsampled to yield the same number of source locations in all subjects (here, 10242 locations per hemisphere). Once the morphing is complete, the data is simply averaged.

What is presented in Figure $\mathbf{1 1}$ is the group average of the dSPM and LCMV beamformer solutions on contrast between faces and scrambled at $170 \mathrm{~ms}$ post-stimulus.

Looking at these results, one can observe that both methods highlight a peak of activation on the right ventral visual cortex known to be involved in face processing (Grill-Spector et al., 2004, 2017; Wakeman and Henson, 2015). To discuss the agreement between these seemingly different methods, we would like to point to Mosher et al. (2003). While LCMV uses an empirical estimate of the data covariance to compute linear 

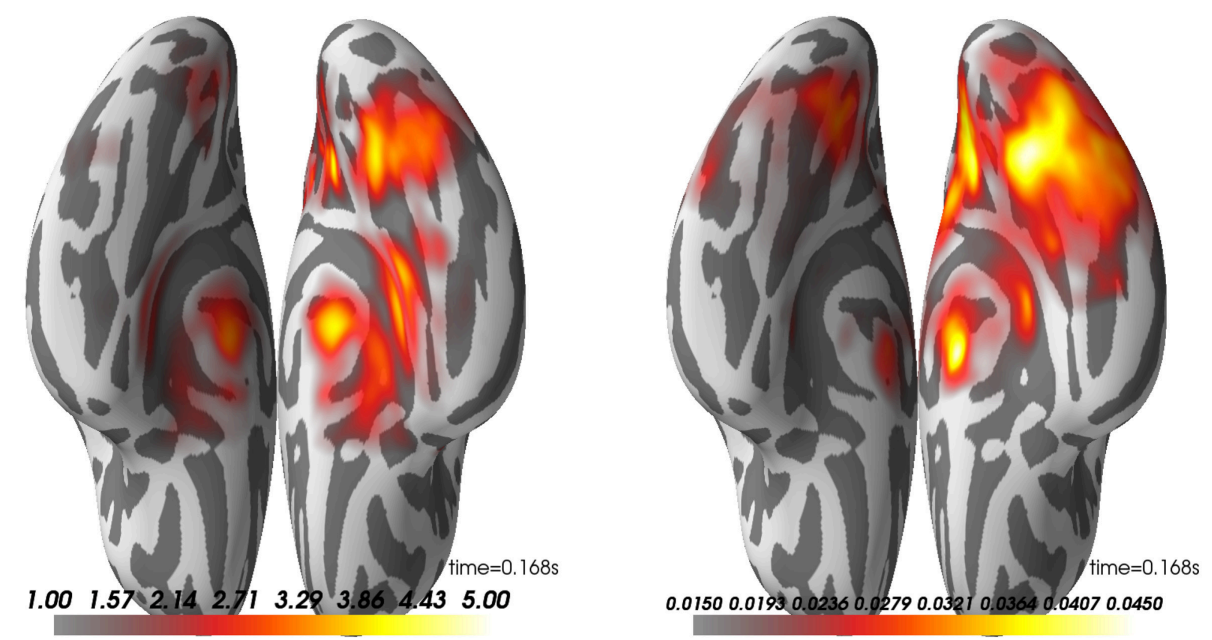

FIGURE 11 | Group average on source reconstruction with dSPM (Left) and LCMV (Right). Here, we have the ventral view of an inflated surface with the anterior-posterior line going from the bottom to top of the image. Right hemisphere is on the right side.

spatial filters, MNE postulates it. It is therefore totally possible that the both inverse methods offer comparable activations on a specific dataset. Note however that we do observe here that the dSPM peak is slightly more anterior.

\subsection{Source-Space Statistics}

Just as we did for the sensor time courses, we can subject the source time courses (here for dSPM only) to a cluster-based permutation test. The null hypothesis is again that there is no significant difference between the data distributions (here measured using cluster size) for faces versus scrambled (paired). Under each permutation, we do a paired t-test across subjects for the difference between the (absolute value of the) faces and scrambled values for each source space vertex and time point. These are clustered, and maximal cluster size for each permutation is selected to form the null distribution. Cluster sizes from the actual data are compared to this null; in this case we find three clusters that lead us to reject the null with $p<0.05$ (see Figure 12).

\subsubsection{Alternatives}

When strong hypotheses exist with regard to spatial, temporal and spectral regions of interest, it may be preferable to test the experimental hypotheses on fewer well-chosen signals. In the context of a group analysis, a linear multilevel modeling approach may provide an interesting option for obtaining joint inference at the single subject and group level (Gelman, 2006; Baayen et al., 2008; Dowding and Haufe, 2017). However, such methods are not yet available in MNE and were therefore not considered in our present analysis. We however, consider that by providing all our analysis code online, such methods could easily be tested and advertised by modifying very little code. Eventually, we hope that such a method will be implemented by an existing or a new MNE contributor.

\section{DISCUSSION AND CONCLUSION}

Analyzing $\mathrm{M} / \mathrm{EEG}$ requires successive operations and transformations on the data. At each analysis stage, the different processing choices can affect the final result in different ways. While this situation encourages tailoring data analysis strategies to the specific demands of the scientific problem, this flexibility comes at a cost and can lead to spurious findings when not handled appropriately (Ioannidis, 2005; Simmons et al., 2011; Carp, 2012a). In the absence of fully automated data analysis pipelines that can optimize the choice of processing steps and parameters, it is crucial to develop principled approaches to planning, conducting, and evaluating M/EEG data analysis.

The present study makes the effort to elucidate common elements and pitfalls of M/EEG analysis. It presents a fully reproducible group analysis of the publicly available dataset from Wakeman and Henson (2015). All code and results are publicly accessible http://mne-tools.github.io/mne-biomaggroup-demo/. The study provides contextualized in-depth discussion of all major steps of the analysis with regard to alternative options, caveats, and quality control measures. As a rare contribution to the M/EEG literature, this study illustrates in comparative figures, the experimental results obtained when changing essential options at different steps in the analysis. In the following, we want to share some insights that we obtained from working together on this study.

\subsection{Collaborative Data Analysis}

In our experience, high-level planning and hands-on data analysis are commonly divided between, e.g., masters or doctoral students, post-docs, and senior researchers. As a consequence, the results are typically appreciated from figures produced without connection to the research code that generated them. In this study, several authors contributed repeatedly to the code, analyses were repeated on different computers, and results 


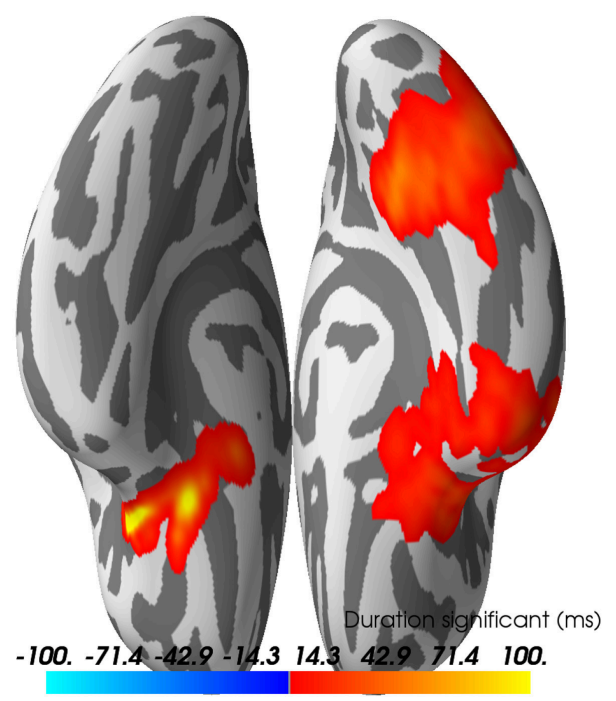

FIGURE 12 | Spatio-temporal source space clusters obtained by nonparametric permutation test that allowed rejection of the null hypothesis that the distribution of data for the "faces" condition was the same as that of "scrambled." The clusters here are collapsed across time such that vertex colors indicate the duration that each vertex was included in its cluster (each cluster here occurring with FWER corrected $p<0.05$ ). Hot colors indicate durations for vertices in clusters where response for faces $>$ scrambled (cool colors would be used for scrambled > faces, but no such clusters were found).

were inspected in an ongoing fashion by many authors. This experience has had as consequence that incoherences, model violations, and other quality concerns were perhaps detected more often than usual, which has greatly contributed to the overall quality of the data analysis. While it is perhaps too extreme or onerous to recommend adopting social interaction habits from open source software development-such as peer review, pair or extreme programming - in scientific data analysis, we believe that data analysis should not be done in isolation. In order to enable full-blown collaborative data analysis in research, analysis must be repeatable, hence, scripted, and a minimum of code organization and readability must be enforced. On the other hand, the best coding efforts will have limited impact if there are not multiple authors with fresh and active data analysis habits. We hope that the example stated by this paper, together with the open source tools and the community it is built upon, can stimulate more collaborative approaches in $\mathrm{M} / \mathrm{EEG}$ research.

\subsection{The Costs of Reproducibility}

It is a commonly neglected reality that reproducibility comes at a price. Making an analysis strictly reproducible not only requires intensified social interactions, hence more time, but also demands more computational resources. It is a combinatorially hard problem if one were to consider all the potential sources of variability. For example, analyses have to be repeated on different computers with different architectures and performance resources. This sometimes reveals differences in results depending on the hardware, operating system, and software packages used. As observed in the past by Glatard et al. (2015), we noticed that some steps in our pipeline such as ICA are more sensitive to these changes, eventually leading to small differences at the end of the pipeline, which is in our case are cluster-level statistics in the source space. Of course, differences due to these changes are harder to control and enforce in the context of today's fast technological progress. Indeed, what we manage to achieve is reproducibility, as opposed to the pure replicability which would be the case if the same results could be achieved even when the computer hardware and software packages were changed.

Also, when code is developed on large desktop computers which is common in many laboratory settings, replication efforts with lower-performance workstations may incur high costs in terms of human processing time. The analysis not only runs slower but may crash, for example due to differences in computer memory resources. We therefore emphasize the responsibility of software developers in providing scalable performance control and the responsibility of hands-on data analysts to design the analysis bearing performance and social constraints in mind. In other words, consider that code needs to run on someone else's computer.

\subsection{When to Stop?}

Obviously, in the light of the current replication crisis, clear rules need to be established on when to stop improving the data analysis (Simmons et al., 2011; Szucs and Ioannidis, 2017). A particular risk is emanating from the possibility of modifying the analysis code to eventually confirm the preferred hypothesis. This would invalidate inference by not acknowledging all the analysis options explored. Similarly, power analysis should be done before conducting the experiment, not afterwards to justify the results (Hoenig and Heisey, 2001). To the best of our knowledge there is no work addressing the estimation of statistical power in the context of MEG/EEG source imaging. This could be an interesting direction for future work. Apart from commonly recommended preregistration practices and clean hold out data systems, we want to emphasize the importance of quality criteria for developing the analysis. The bulk of M/EEG preprocessing tasks are either implicitly or explicitly model-based, as shown by the rich battery of quality control visualizations presented in this manuscript. Such plots allow to assess if M/EEG analysis outputs can be considered good signals. Consequently, analysis should be stopped when no further improvement on quality control metrics is to be expected, within a reasonable time investment. In other words, not research hypotheses (and statistical significance of results) but rather signal quality metrics are the criterion for constructing M/EEG analyses. Ideally, only when quality control is done, should the contrast(s) of interest be investigated.

With these broader insights in mind, we will make an attempt to extract from our analysis practical recommendations that should facilitate future M/EEG analyses. We encourage the reader not to take the analysis presented here as a direct justifications for parameter choices used in their analyses, but instead learn the principles underlying the choices made in our examples. The general rule is: assess your options and chose the optimal measure 
at each processing step, then visualize and automate as much as you can.

Practical recommendations:

1. Know your I/O. Make sure to have a clear idea about the metadata available in your recordings and that the software package knows about relevant auxiliary channels, e.g, stim, EOG, ECG. Use custom MNE functions and other libraries to add quick reading support if $\mathrm{I} / \mathrm{O}$ for a file-type is not readily supported.

2. Understand the origin of the noise. Inspect your raw data and power spectra to see if and how much denoising is necessary. When using methods such as SSS, SSP, ICA, or reference-channel correction, be aware of their implications for later processing. Remember also to process your empty room data the same way. The interpretation of sensor types may change. Denoising may implicitly act as a high-pass filter (cf. tSSS). High-pass filtering or baselining may not be ideal, depending on the paradigm. For calibrating your inverse solution, think of what is an appropriate noise model, it may be intrinsically linked to your hypothesis.

3. Mind signals of non-interest. Detect and visualize your physiological artifacts, e.g. ECG, EOG, prior to attempting to mitigate them. Choose an option that is precise enough for your data. There is no absolute removal, only changes in signal-to-noise ratio. Not explicitly suppressing any artifacts may also be a viable option in some situations, whereas a downstream method (e.g., temporal decoding) will not benefit from them. When employing an artifact removal technique, visualize how much of your signal of interest is discarded.

4. Visually inspect at multiple stages. Use diagnostic visualizations often to get a sense of signal characteristics, from noise sources, to potential signals of interest. Utilize knowledge of paradigms (e.g., existence of an N100 response) to validate steps. Visual inspection of data quality and SNR is recommended even if the processing is automated. When using the an anatomical pipeline, look at your coregistration and head models to make sure they are satisfactory. Small errors can propagate and induce spurious results. Check for model violations when working with inverse solvers and understand them. Inappropriate noise models will distort your estimated sources in simple or complex ways and may give rise to spurious effects.

5. Apply statistics in a planned way. Averaging data is a type of statistical transformation. Make sure that what you average is actually comparable. To handle the multiple-comparisons problem, different options exist. Non-parametric hypothesistests with clustering and multivariate decoding are two such options, and they are not mutually exclusive. Keep in mind that MEEG is primarily about time, not space. A whole-brain approach may or may not be the best thing to pursue in your situation. Anatomical labels may provide an effective way of reducing the statistical search space.

6. Be mindful of non-deterministic steps. To maximize reproducibility, make sure to fix the random initialization of non-deterministic algorithms such as ICA. Not only does it ensure reproducibility, debugging is also easier when the code is deterministic. Prefer automated scripts as opposed to interactive or manual pipelines wherever possible.

7. Keep software versions fixed. In an ideal world, software (and hardware) versions would not matter, as each operation necessary for data analysis should be tested against known results to ensure consistency across platforms and versions. However, this ideal cannot always be met in practice. To limit difficulties, do not change software versions, hardware or operating system versions in the middle of your analysis. Keep in mind that MNE is based on several other pieces of software. Updating them can have an impact on the outcome of MNE routines. Once data analysis is complete, cross-checking on different platforms or with different software versions can be useful for community feedback and identifying fragile or problematic steps.

Finally, we would like to emphasize once more that there is no single recipe for all data. Indeed, this approach can be dangerous and lead to surprising results. In that sense, there might be other equally valid pipelines recommended by other academic softwares (Brunet et al., 2011; Dalal et al., 2011; Litvak et al., 2011; Oostenveld et al., 2011; Tadel et al., 2011). The aim of this paper is to help the reader assess the right alternative at each stage of the analysis rather than recommend a single pipeline.

In order to facilitate the reproduction of all the results presented in this manuscript, all the code used to make the figures in this paper, but also much more, is available at http://mne-tools. github.io/mne-biomag-group-demo/.

\section{AUTHOR CONTRIBUTIONS}

All authors contributed extensively to the manuscript. MJ, EL, $\mathrm{DE}, \mathrm{JL}$, and AG conducted experiments, wrote code to analyze the data, interpreted the data, and wrote the manuscript. ST and $\mathrm{MH}$ interpreted the data, participated in the scientific discussions, and provided critical insights. All authors reviewed the manuscript and approved it for publication.

\section{ACKNOWLEDGMENTS}

We would like to thank the many members of the MNE community who have contributed through code, comments, and even complaints, to the improvement and design of this software.

During the preparation of this manuscript, we benefited greatly from discussions with other software package maintainers, such as Brainstorm maintainers who suggested that we visualize PSDs on a channel-by-channel basis to more effectively identify bad sensors. We thank Sami Aboud for discussions on the manuscript and proof-reading.

This work was supported by National Institute of Biomedical Imaging and Bioengineering grants 5R01EB009048, NIH R01 MH106174 and P41RR014075, National Institute on Deafness and Other Communication Disorders fellowship F32DC012456, NSF awards 0958669 and 1042134, the French National Research Agency (ANR-14-NEUC-0002-01) and the European Research Council Starting Grant SLAB ERC-YStG-676943. 


\section{REFERENCES}

Acunzo, D. J., MacKenzie, G., and van Rossum, M. C. (2012). Systematic biases in early ERP and ERF components as a result of high-pass filtering. J. Neurosci. Methods 209, 212-218. doi: 10.1016/j.jneumeth.2012.06.011

Baayen, R. H., Davidson, D. J., and Bates, D. M. (2008). Mixed-effects modeling with crossed random effects for subjects and items. J. Mem. Lang. 59, 390-412. doi: 10.1016/j.jml.2007.12.005

Baillet, S. (2017). Magnetoencephalography for brain electrophysiology and imaging. Nat. Neurosci. 20, 327-339. doi: 10.1038/nn.4504

Bigdely-Shamlo, N., Kreutz-Delgado, K., Robbins, K., Miyakoshi, M., Westerfield, M., Bel-Bahar, T., et al. (2013). "Hierarchical event descriptor (HED) tags for analysis of event-related EEG studies," in Global Conference on Signal and Information Processing (GlobalSIP) (IEEE) (Austin, TX), 1-4.

Brunet, D., Murray, M. M., and Michel, C. M. (2011). Spatiotemporal analysis of multichannel EEG: Cartool. Comput. Intell. Neurosci. 2011:813870. doi: $10.1155 / 2011 / 813870$

Button, K. S., Ioannidis, J. P., Mokrysz, C., Nosek, B. A., Flint, J., Robinson, E. S., et al. (2013). Power failure: why small sample size undermines the reliability of neuroscience. Nat. Rev. Neurosci. 14, 365-376. doi: 10.1038/nrn3475

Carp, J. (2012a). On the plurality of (methodological) worlds: estimating the analytic flexibility of fMRI experiments. Front. Neurosci. 6:149. doi: $10.3389 /$ fnins. 2012.00149

Carp, J. (2012b). The secret lives of experiments: methods reporting in the fMRI literature. Neuroimage 63, 289-300. doi: 10.1016/j.neuroimage.2012.07.004

Dalal, S. S., Zumer, J. M., Guggisberg, A. G., Trumpis, M., Wong, D. D., Sekihara, K., et al. (2011). MEG/EEG source reconstruction, statistical evaluation, and visualization with NUTMEG. Comput. Intell. Neurosci. 2011:758973. doi: 10.1155/2011/758973

Dale, A. M., Fischl, B., and Sereno, M. I. (1999). Cortical surface-based analysis I: Segmentation and surface reconstruction. Neuroimage 9, 179-194.

Dale, A., Liu, A., Fischl, B., and Buckner, R. (2000). Dynamic statistical parametric mapping: combining $\mathrm{fMRI}$ and MEG for high-resolution imaging of cortical activity. Neuron 26, 55-67. doi: 10.1016/S0896-6273(00)81138-1

Dammers, J., Schiek, M., Boers, F., Silex, C., Zvyagintsev, M., Pietrzyk, U., et al. (2008). Integration of amplitude and phase statistics for complete artifact removal in independent components of neuromagnetic recordings. IEEE Trans. Biomed. Eng. 55, 2353-2362. doi: 10.1109/TBME.2008.926677

Delorme, A., and Makeig, S. (2004). EEGLAB: an open source toolbox for analysis of single-trial EEG dynamics including independent component analysis. J. Neurosci. Methods 134, 9-21. doi: 10.1016/j.jneumeth.2003.10.009

Delorme, A., Miyakoshi, M., Jung, T.-P., and Makeig, S. (2015). Grand average ERP-image plotting and statistics: a method for comparing variability in eventrelated single-trial eeg activities across subjects and conditions. J. Neurosci. Methods 250(Suppl. C), 3-6. doi: 10.1016/j.jneumeth.2014.10.003

Delorme, A., Mullen, T., Kothe, C., Acar, Z., Bigdely-Shamlo, N., Vankov, A., et al. (2011). EEGLAB, SIFT, NFT, BCILAB, and ERICA: new tools for advanced EEG processing. Intell. Neurosci. 2011:130714. doi: 10.1155/2011/130714

Dowding, I., and Haufe, S. (2017). Statistical inference for nested data using sufficient summary statistics: a tutorial. ArXiv e-prints.

Engemann, D., Strohmeier, D., Larson, E., and Gramfort, A. (2015). "Mind the noise covariance when localizing brain sources with $\mathrm{m} / \mathrm{EEG}$," in 2015 International Workshop on Pattern Recognition in NeuroImaging (PRNI) (Stanford, CA), 9-12.

Engemann, D. A., and Gramfort, A. (2015). Automated model selection in covariance estimation and spatial whitening of MEG and EEG signals. Neuroimage 108, 328-342. doi: 10.1016/j.neuroimage.2014.12.040

Fischl, B., Sereno, M., and Dale, A. (1999). Cortical surface-based analysis II: inflation, flattening, and a surface-based coordinate system. Neuroimage 9, 195-207.

Gelman, A. (2006). Multilevel (hierarchical) modeling: what it can and cannot do. Technometrics 48, 432-435. doi: 10.1198/004017005000000661

Genovese, C. R., Lazar, N. A., and Nichols, T. (2002). Thresholding of statistical maps in functional neuroimaging using the false discovery rate. Neuroimage 15, 870-878. doi: 10.1006/nimg.2001.1037

Glatard, T., Lewis, L. B., Ferreira da Silva, R., Adalat, R., Beck, N., Lepage, C., et al. (2015). Reproducibility of neuroimaging analyses across operating systems. Front. Neuroinformatics 9:12. doi: 10.3389/fninf.2015.00012
Gorgolewski, K. J., Auer, T., Calhoun, V. D., Craddock, R. C., Das, S., Duff, E. P., et al. (2016). The brain imaging data structure, a format for organizing and describing outputs of neuroimaging experiments. Sci. Data 3:160044. doi: $10.1038 /$ sdata.2016.44

Götz, T., Milde, T., Curio, G., Debener, S., Lehmann, T., Leistritz, L., et al. (2015). Primary somatosensory contextual modulation is encoded by oscillation frequency change. Clin. Neurophysiol.126, 1769-1779. doi: 10.1016/j.clinph.2014.12.028

Gramfort, A., Keriven, R., and Clerc, M. (2010). Graph-based variability estimation in single-trial event-related neural responses. IEEE Trans. Biomed. Eng. 57, 1051-1061. doi: 10.1109/TBME.2009.2037139

Gramfort, A., Luessi, M., Larson, E., Engemann, D. A., Strohmeier, D., Brodbeck, C., et al. (2013a). MEG and EEG data analysis with MNE-Python. Front. Neurosci. 7:267. doi: 10.3389/fnins.2013.00267

Gramfort, A., Luessi, M., Larson, E., Engemann, D. A., Strohmeier, D., Brodbeck, C., et al. (2014). MNE software for processing MEG and EEG data. Neuroimage 86, 446-460. doi: 10.1016/j.neuroimage.2013.10.027

Gramfort, A., Strohmeier, D., Haueisen, J., Hämäläinen, M. S., and Kowalski, M. (2013b). Time-frequency mixed-norm estimates: Sparse M/EEG imaging with non-stationary source activations. Neuroimage 70, 410-422. doi: 10.1016/j.neuroimage.2012.12.051

Grill-Spector, K., Knouf, N., and Kanwisher, N. (2004). The fusiform face area subserves face perception, not generic within-category identification. Nat. Neurosci. 7, 555-562. doi: 10.1038/nn1224

Grill-Spector, K., Weiner, K. S., Kay, K., and Gomez, J. (2017). The functional neuroanatomy of human face perception. Annu. Rev. Vision Sci. 3, 167-196. doi: 10.1146/annurev-vision-102016-061214

Gross, J., Baillet, S., Barnes, G. R., Henson, R. N., Hillebrand, A., Jensen, O., et al. (2013). Good practice for conducting and reporting MEG research. Neuroimage 65, 349-363. doi: 10.1016/j.neuroimage.2012.10.001

Gross, J., Kujala, J., Hämäläinen, M., and Timmermann, L. (2001). Dynamic imaging of coherent sources: studying neural interactions in the human brain. Proc. Natl. Acad. Sci. U.S.A. 98, 694-699. doi: 10.1073/pnas.98.2.694

Hämäläinen, M., and Ilmoniemi, R. (1984). Interpreting Magnetic Fields of the Brain: Minimum Norm Estimates. Technical Report TKK-F-A559, Helsinki University of Technology.

Haufe, S., Meinecke, F., Görgen, K., Dähne, S., Haynes, J.-D., Blankertz, B., et al. (2014). On the interpretation of weight vectors of linear models in multivariate neuroimaging. Neuroimage 87, 96-110. doi: 10.1016/j.neuroimage.2013. 10.067

Hauk, O., Wakeman, D. G., and Henson, R. (2011). Comparison of noise-normalized minimum norm estimates for MEG analysis using multiple resolution metrics. Neuroimage 54, 1966-1974. doi: 10.1016/j.neuroimage.2010.09.053

Hoenig, J. M. and Heisey, D. M. (2001). The abuse of power: the pervasive fallacy of power calculations for data analysis. Am. Statist. 55, 19-24.

Hyvarinen, A. (1999). Fast and robust fixed-point algorithms for independent component analysis. IEEE Trans. Neural Netw. 10, 626-634.

Ifeachor, E. C., and Jervis, B. W. (2002). Digital Signal Processing: A Practical Approach. Pearson Education.

Ioannidis, J. P. (2005). Why most published research findings are false. PLoS Med. 2:e124. doi: 10.1371/journal.pmed.0020124

Jas, M., Engemann, D. A., Bekhti, Y., Raimondo, F., and Gramfort, A. (2017). Autoreject: automated artifact rejection for MEG and EEG data. Neuroimage 159, 417-429. doi: 10.1016/j.neuroimage.2017.06.030

Jung, T.-P., Humphries, C., Lee, T.-W., Makeig, S., McKeown, M. J., Iragui, V., et al. (1998). "Extended ICA removes artifacts from electroencephalographic recordings," in Advances in Neural Information Processing Systems 10 eds M. I. Jordan, M. J. Kearns, and S. A. Solla (Denver, CO: MIT Press), 894-900.

Khan, S., and Cohen, D. (2013). Note: magnetic noise from the inner wall of a magnetically shielded room. Rev. Sci. Instrum. 84:056101. doi: $10.1063 / 1.4802845$

King, J. R., and Dehaene, S. (2014). Characterizing the dynamics of mental representations: the temporal generalization method. Trends Cogn Sci. 18, 203-210. doi: 10.1016/j.tics.2014.01.002

Kriegeskorte, N., Mur, M., and Bandettini, P. (2008). Representational similarity analysis-connecting the branches of systems neuroscience. Front. Syst. Neurosci. 2:4. doi: 10.3389/neuro.06.004.2008 
Larson, E., and Lee, A. K. C. (2013). The cortical dynamics underlying effective switching of auditory spatial attention. Neuroimage 64(Suppl. C), 365-370. doi: 10.1016/j.neuroimage.2012.09.006

Lin, F.-H., Witzel, T., Ahlfors, S. P., Stufflebeam, S. M., Belliveau, J. W., and Hämäläinen, M. S. (2006). Assessing and improving the spatial accuracy in MEG source localization by depth-weighted minimum-norm estimates. Neuroimage 31, 160-171. doi: 10.1016/j.neuroimage.2005.11.054

Litvak, V., Mattout, J., Kiebel, S., Phillips, C., Henson, R., Kilner, J., et al. (2011). EEG and MEG data analysis in SPM8. Comput. Intell. Neurosci. 2011:852961. doi: $10.1155 / 2011 / 852961$

Maess, B., Schröger, E., and Widmann, A. (2016). High-pass filters and baseline correction in M/EEG analysis-continued discussion. J. Neurosci. Methods 266, 171. doi: 10.1016/j.jneumeth.2016.01.016

Maris, E., and Oostenveld, R. (2007). Nonparametric statistical testing of EEG- and MEG-data. J. Neurosci. Methods 164, 177-190. doi: 10.1016/j.jneumeth.2007.03.024

Mosher, J. C., Lewis, P. S., and Leahy, R. M. (1992). Multiple dipole modeling and localization from spatio-temporal MEG data. IEEE Trans. Biomed. Eng. 39, 541-553.

Mosher, J. C., Baillet, S., and Leahy, R. M. (2003). "Equivalence of linear approaches in bioelectromagnetic inverse solutions," in 2003 IEEE Workshop on Statistical Signal Processing (St. Louis, MI), 294-297.

Mosher, J. C., Leahy, R. M., and Lewis, P. S. (1999). EEG and MEG: forward solutions for inverse methods. IEEE Trans. Biomed. Eng. 46, 245-259.

Niso, G., Gorgolewski, K. J., Bock, E., Brooks, T. L., Flandin, G., Gramfort, A., et al. (2018). MEG-BIDS, the brain imaging data structure extended to magnetoencephalography. Sci. Data 5:180110. doi: 10.1038/sdata.2018.110

Oostenveld, R., Fries, P., Maris, E., and Schoffelen, J.-M. (2011). FieldTrip: Open source software for advanced analysis of MEG, EEG, and invasive electrophysiological data. Comput. Intell. Neurosci. 2011:156869. doi: 10.1155/2011/156869

Parks, T. W., and Burrus, C. S. (1987). Digital Filter Design. New York, NY: Wiley-Interscience.

Percival, D. B., and Walden, A. T. (1993). Spectral Analysis for Physical Applications. Cambridge, UK: Cambridge University Press.

Perrin, F., Pernier, J., Bertrand, O., and Echallier, J. (1989). Spherical splines for scalp potential and current density mapping. Electroencephalogr. Clin. Neurophysiol. 72, 184-187.

Poldrack, R. A., and Gorgolewski, K. J. (2017). OpenfMRI: open sharing of task fMRI data. Neuroimage 144:259-261. doi: 10.1016/j.neuroimage.2015.05.073

Ramkumar, P., Jas, M., Pannasch, S., Hari, R., and Parkkonen, L. (2013). Featurespecific information processing precedes concerted activation in human visual cortex. J. Neurosci. 33, 7691-7699. doi: 10.1523/JNEUROSCI.3905-12.2013

Rivet, B., Souloumiac, A., Attina, V., and Gibert, G. (2009). xDAWN algorithm to enhance evoked potentials: application to brain-computer interface. IEEE Trans. Biomed. Eng. 56, 2035-2043. doi: 10.1109/TBME.2009.2012869

Rousselet, G. A. (2012). Does filtering preclude us from studying ERP timecourses? Front. Psychol. 3:131. doi: 10.3389/fpsyg.2012.00131

Scherg, M., and Von Cramon, D. (1985). Two bilateral sources of the late AEP as identified by a spatio-temporal dipole model. Electroencephalogr. Clin. Neurophysiol. 62, 32-44.

Schurger, A., Marti, S., and Dehaene, S. (2013). Reducing multi-sensor data to a single time course that reveals experimental effects. BMC Neurosci. 14:122. doi: 10.1186/1471-2202-14-122

Sekihara, K., Sahani, M., and Nagarajan, S. S. (2005). Localization bias and spatial resolution of adaptive and non-adaptive spatial filters for MEG source reconstruction. Neuroimage 25, 1056-1067. doi: 10.1016/j.neuroimage.2004.11.051

Simmons, J., Nelson, L., and Simonsohn, U. (2011). False-positive psychology: undisclosed flexibility in data collection and analysis allows presenting anything as significant. Psychol. Sci. 22, 1359-1366. doi: $10.1177 / 0956797611417632$
Slepian, D. (1978). Prolate spheroidal wave functions, Fourier analysis, and uncertainty: The discrete case. Bell Syst. Tech. J. 57, 1371-1430.

Smith, N. J., and Kutas, M. (2015a). Regression-based estimation of ERP waveforms: I. the rERP framework. Psychophysiology 52, 157-168. doi: $10.1111 /$ psyp. 12317

Smith, N. J., and Kutas, M. (2015b). Regression-based estimation of ERP waveforms: II. nonlinear effects, overlap correction, and practical considerations. Psychophysiology 52, 169-181. doi: 10.1111/psyp.12320

Smith, S. M., and Nichols, T. E. (2009). Threshold-free cluster enhancement: addressing problems of smoothing, threshold dependence and localisation in cluster inference. Neuroimage 44, 83-98. doi: 10.1016/j.neuroimage.2008.03.061

Szucs, D., and Ioannidis, J. (2017). Empirical assessment of published effect sizes and power in the recent cognitive neuroscience and psychology literature. PLoS Biol. 15:e2000797. doi: 10.1371/journal.pbio.2000797

Tadel, F., Baillet, S., Mosher, J. C., Pantazis, D., and Leahy, R. M. (2011). Brainstorm: a user-friendly application for MEG/EEG analysis. Comput. Intell. Neurosci. 2011:879716. doi: 10.1155/2011/879716

Tanner, D., Morgan-Short, K., and Luck, S. J. (2015). How inappropriate high-pass filters can produce artifactual effects and incorrect conclusions in ERP studies of language and cognition. Psychophysiology 52, 997-1009. doi: $10.1111 /$ psyp. 12437

Taulu, S. (2006). Spatiotemporal Signal Space Separation method for rejecting nearby interference in MEG measurements. Phys. Med. Biol. 51, 1759-1769. doi: 10.1088/0031-9155/51/7/008

Uusitalo, M., and Ilmoniemi, R. (1997). Signal-space projection method for separating MEG or EEG into components. Med. Biol. Eng. Comput. 35, 135140.

Van Veen, B., van Drongelen, W., Yuchtman, M., and Suzuki, A. (1997). Localization of brain electrical activity via linearly constrained minimum variance spatial filtering. IEEE Trans. Biomed. Eng. 44, 867-580.

Viola, F. C., Thorne, J., Edmonds, B., Schneider, T., Eichele, T., and Debener, S. (2009). Semi-automatic identification of independent components representing EEG artifact. Clin. Neurophysiol. 120, 868-877. doi: 10.1016/j.clinph.2009.01.015

Wakeman, D. G., and Henson, R. N. (2015). A multi-subject, multimodal human neuroimaging dataset. Sci. Data 2. doi: 10.1038/sdata. 2015.1

Welch, P. (1967). The use of fast Fourier transform for the estimation of power spectra: a method based on time averaging over short, modified periodograms. IEEE Trans. Audio Electroacoust. 15, 70-73.

Widmann, A. and Schröger, E. (2012). Filter effects and filter artifacts in the analysis of electrophysiological data. Front. Psychol. 3:233. doi: 10.3389/fpsyg.2012.00233

Widmann, A., Schröger, E., and Maess, B. (2015). Digital filter design for electrophysiological data-a practical approach. J. Neurosci. Methods 250, 34-46. doi: 10.1016/j.jneumeth.2014.08.002

Woolrich, M., Hunt, L., Groves, A., and Barnes, G. (2011). MEG beamforming using Bayesian PCA for adaptive data covariance matrix regularization. Neuroimage 57, 1466-1479. doi: 10.1016/j.neuroimage.2011.04.041

Conflict of Interest Statement: The authors declare that the research was conducted in the absence of any commercial or financial relationships that could be construed as a potential conflict of interest.

Copyright (c) 2018 Jas, Larson, Engemann, Leppäkangas, Taulu, Hämäläinen and Gramfort. This is an open-access article distributed under the terms of the Creative Commons Attribution License (CC BY). The use, distribution or reproduction in other forums is permitted, provided the original author(s) and the copyright owner(s) are credited and that the original publication in this journal is cited, in accordance with accepted academic practice. No use, distribution or reproduction is permitted which does not comply with these terms. 